\title{
Copper(II)-Catalyzed Ether Synthesis from Aliphatic Alcohols and Potassium Organotrifluoroborate Salts
}

\author{
Tan D. Quach and Robert A. Batey.* \\ Davenport Research Laboratories, Department of Chemistry, University of Toronto, \\ 80 St. George Street, Toronto, Ontario, M5S 3H6, Canada \\ rbatey@chem.utoronto.ca
}

\section{General Synthetic Methods}

$\mathrm{CH}_{2} \mathrm{Cl}_{2}$ was distilled from $\mathrm{CaH}_{2}$ under argon. All other commercial solvents and reagents were used as received from the Aldrich Chemical Company, Fischer Scientific Ltd., Strem or BDH. All glassware was oven dried at $210^{\circ} \mathrm{C}$ and allowed to cool under a stream of dry nitrogen.

References following compound names indicate literature articles where ${ }^{1} \mathrm{H}$ and ${ }^{13} \mathrm{C}$ NMR data have previously been reported.

Silica gel (60 ̊, 230-400 mesh) used in flash column chromatography was obtained from Whatman Company, Toronto Research Chemicals, Inc. or Silicycle, and was used as received. Analytical thin-layer chromatography (TLC) was performed on pre-coated silica gel plates (Ultra Pure Silica Gel Plates purchased from Silicycle), visualized with a Spectroline $\mathrm{UV}_{254}$ lamp, and stained with a $20 \%$ phosphomolybdic acid in ethanol solution. Solvent systems associated with $\mathrm{R}_{f}$ values and flash column chromatography are reported as v/v ratios.

Melting points were obtained using a Fisher-Johns melting point apparatus, and are uncorrected. Specific optical rotations were determined on a Perkin-Elmer 243B Polarimeter under the conditions indicated using the sodium D line (589 nm). Sample concentrations are reported as grams per $100 \mathrm{~mL}$ solvent. Analytical chiral HPLC (of 2t) was performed on a Chiralcel OD column (4.6 x $250 \mathrm{~mm})$ obtained from Daicel Chemical Industries, Ltd. ${ }^{1} \mathrm{H}$ and ${ }^{13} \mathrm{C}$ NMR were recorded at 400 or $300 \mathrm{MHz}$ and 100 or $75 \mathrm{MHz}$ respectively on a Varian Unity 400, Gemini 300 or Mercury 300 spectrometer. Proton chemical shifts were internally referenced to the residual proton resonance in $\mathrm{CDCl}_{3}(\delta 7.26 \mathrm{ppm})$. Carbon chemical shifts were internally referenced to the dueterated solvent signals in $\mathrm{CDCl}_{3}(\delta 77.20 \mathrm{ppm})$. FT-IR spectra were recorded on a Perkin-Elmer Spectrum 1000 spectrometer with samples loaded as neat films on $\mathrm{NaCl}$ plates or as pressed $\mathrm{KBr}$ discs. Low and high resolution mass spectra were recorded on a Bell and Howell 21-490 spectrometer and an AEI MS3074 spectrometer respectively. 


\section{Synthetic Methods and Characterization Data}

\section{Representative Procedure for the Cross-Coupling of $\mathrm{PhBF}_{3}{ }^{-} \mathrm{K}^{+}$with Aliphatic Alcohols}

A suspension of $\mathrm{PhBF}_{3}{ }^{-} \mathrm{K}^{+}(0.736 \mathrm{~g}, 4.00 \mathrm{mmol}), \mathrm{Cu}(\mathrm{OAc})_{2} \cdot \mathrm{H}_{2} \mathrm{O}(0.040 \mathrm{~g}, 0.20 \mathrm{mmol})$, DMAP (0.050 g, $0.40 \mathrm{mmol})$ and powdered $4 \AA$ molecular sieves $(1.50 \mathrm{~g})$ in $\mathrm{CH}_{2} \mathrm{Cl}_{2}(15.0$ $\mathrm{mL}$ ) was stirred for 5 minutes at room temperature. To this stirring suspension was added $(E)$-cinnamyl alcohol $(0.268 \mathrm{~g}, 2.00 \mathrm{mmol})$. The stirring reaction mixture was then sealed with a rubber septa and stirred under an atmosphere of oxygen (delivered via an oxygen tank connected to a manifold). Following a period of $24 \mathrm{~h}$, the crude reaction mixture was filtered through a plug of celite to remove the molecular sieves and any insoluble byproducts, and then concentrated in vacuo to afford the crude product mixture. The product (2a) was isolated by silica gel column chromatography (eluting with hexanes:EtOAc 9:1 3:1 gradient) as a white crystalline solid in $89 \%$ yield $(0.374 \mathrm{~g}$, $1.78 \mathrm{mmol})$.

\section{Products of Experiments Summarized in Table 1}

(E)-Cinnamylphenyl ether (2a) (Keinan, E.; Sahai, M.; Roth, Z. J. Org. Chem. 1985, 50, 3558-3566.)

Isolated as a white, crystalline solid in $89 \%$ yield: $\mathrm{R}=0.6\left(9: 1\right.$ hexanes:EtOAc); ${ }^{1} \mathrm{H}$ NMR $\left(\mathrm{CDCl}_{3}, 300 \mathrm{MHz}\right) \delta 7.48(2 \mathrm{H}, \mathrm{d}, J=7.0 \mathrm{~Hz}), 7.44-7.28(5 \mathrm{H}, \mathrm{m}), 7.11$ - $6.95(3 \mathrm{H}$, m), $6.80(1 \mathrm{H}, \mathrm{d}, J=16.0 \mathrm{~Hz}), 6.48(1 \mathrm{H}, \mathrm{dt}, J=16.0,6.0 \mathrm{~Hz}), 4.75(2 \mathrm{H}, \mathrm{dd}, J=6.0,1.5$ $\mathrm{Hz}) ;{ }^{13} \mathrm{C} \mathrm{NMR}\left(\mathrm{CDCl}_{3}, 75 \mathrm{MHz}\right) \delta 158.66,136.54,133.05,129.58,128.67,127.97$, $126.67,124.63,121.00,114.91,68.76$.

\section{Phenyl-(3-phenylpropyl) ether (2b)}

Isolated as a light yellow oil in $95 \%$ yield: $\mathrm{R}_{f}=0.7$ (9:1 hexanes:EtOAc); ${ }^{1} \mathrm{H} \mathrm{NMR}$ $\left(\mathrm{CDCl}_{3}, 300 \mathrm{MHz}\right) \delta 7.40-7.24(7 \mathrm{H}, \mathrm{m}), 7.04-6.94(3 \mathrm{H}, \mathrm{m}), 4.03(2 \mathrm{H}, \mathrm{t}, J=6.5 \mathrm{~Hz})$, $2.90(2 \mathrm{H}, \mathrm{t}, J=7.5 \mathrm{~Hz}), 2.22-2.14(2 \mathrm{H}, \mathrm{m}) ;{ }^{13} \mathrm{C} \mathrm{NMR}\left(\mathrm{CDCl}_{3}, 75 \mathrm{MHz}\right) \delta 158.87$, $141.45,129.38,128.48,128.37,125.89,120.57,114.54,66.93,32.49,31.19$; IR (film) $v$ 3062, 3027, 2946, 2870, 1736, 1600, 1586, 1497, 1470, 1454, 1389, 1301, 1246, 1172, 1080, 1039, 940, 882, 806, $752 \mathrm{~cm}^{-1}$; LRMS (EI): $\mathrm{m} / z=213$ (12), 212 (67), 119 (21), 118 (80), 117 (44), 94 (22), 92 (12), 91 (100), 77 (13), 65 (21); HRMS (EI): m/z calcd. for $\left(\mathrm{C}_{15} \mathrm{H}_{16} \mathrm{O}^{+}\right)=212.1201$, found $=212.1206$. 
Phenylpropyl ether (2c) (Chen, C. T.; Marder, S. R.; Cheng, L. T. J. Amer. Chem. Soc. 1994, 116, 3117-3118.)

Isolated as a clear liquid in $78 \%$ yield: $\mathrm{R}_{f}=0.8$ (9:1 hexanes:EtOAc); ${ }^{1} \mathrm{H}$ NMR $\left(\mathrm{CDCl}_{3}\right.$, $300 \mathrm{MHz}) \delta 7.40-7.28(2 \mathrm{H}, \mathrm{m}), 7.00-6.90(3 \mathrm{H}, \mathrm{m}), 3.95(2 \mathrm{H}, \mathrm{t}, J=7.0 \mathrm{~Hz}), 1.85(2 \mathrm{H}$, sextet, $J=7.0 \mathrm{~Hz}), 1.09(3 \mathrm{H}, \mathrm{t}, J=7.0 \mathrm{~Hz}) ;{ }^{13} \mathrm{C} \mathrm{NMR}\left(\mathrm{CDCl}_{3}, 75 \mathrm{MHz}\right) \delta 159.01$, $129.37,120.47,114.53,69.57,23.00,10.96$.

Benzylphenyl ether (2d) (Forrester, J.; Jones, R. V. H.; Newton, L.; Preston, P. N. Tetrahedron 2001, 57, 2871-2884.)

Isolated as a white, crystalline solid in $71 \%$ yield: $\mathrm{R}=0.7$ (9:1 hexanes:EtOAc); ${ }^{1} \mathrm{H}$ NMR $\left(\mathrm{CDCl}_{3}, 300 \mathrm{MHz}\right) \delta 7.47-7.25(7 \mathrm{H}, \mathrm{m}), 7.02-6.93(3 \mathrm{H}, \mathrm{m}), 5.09(2 \mathrm{H}, \mathrm{s}) ;{ }^{13} \mathrm{C}$ NMR $\left(\mathrm{CDCl}_{3}, 75 \mathrm{MHz}\right) \delta 158.67,137.05,129.45,128.56,127.91,127.46,120.95$, $114.90,70.12$.

\section{(4-Bromobenzyl)phenyl ether (2e)}

Isolated as a white, crystalline solid in $94 \%$ yield: $\mathrm{R}_{\mathrm{f}}=0.5$ (3:1 hexanes:toluene); $\mathrm{mp}=$ $91-92{ }^{\circ} \mathrm{C}\left(\mathrm{CH}_{2} \mathrm{Cl}_{2}\right) ;{ }^{1} \mathrm{H} \mathrm{NMR}\left(\mathrm{CDCl}_{3}, 300 \mathrm{MHz}\right) \delta 7.52(2 \mathrm{H}, \mathrm{d}, J=8.0 \mathrm{~Hz}), 7.34-7.25$ (4H, m), 7.02 - $6.94(3 \mathrm{H}, \mathrm{m}), 5.03(2 \mathrm{H}, \mathrm{s}) ;{ }^{13} \mathrm{C} \mathrm{NMR}(\mathrm{CDCl}, 75 \mathrm{MHz}) \delta 158.36,136.07$, 131.67, 129.52, 129.04, 121.18, 121.18, 114.87, 69.34; IR (film) v 3080, 3048, 2922, 2878, 1916, 1600, 1490, 1456, 1406, 1384, 1240, 1079, 1032, 1016, 994, 871, 811, 753, $691 \mathrm{~cm}^{-1}$; LRMS (EI): $\mathrm{m} / z=264$ (21), 262 (22), 172 (12), 171 (98), 170 (14), 169 (100), 90 (30), 89 (19), 65 (9), 63 (10), 51 (6); HRMS (EI): m/z calcd. for $\left(\mathrm{C}_{13} \mathrm{H}_{11} \mathrm{BrO}^{+}\right)=$ 261.9993 , found $=261.9994$.

(4-Nitrobenzyl)phenyl ether (2f) (Maslak, P.; Guthrie, R. D. J. Am. Chem. Soc. 1986, 108, 2628-2636.)

Isolated as a white, crystalline solid in $75 \%$ yield: $\mathrm{R}_{f}=0.3$ (9:1 hexanes:toluene); ${ }^{1} \mathrm{H}$ NMR $(\mathrm{CDCl}, 300 \mathrm{MHz}) \delta 8.23(2 \mathrm{H}, \mathrm{d}, J=8.5 \mathrm{~Hz}), 7.61(2 \mathrm{H}, \mathrm{d}, J=8.5 \mathrm{~Hz}), 7.32(2 \mathrm{H}, \mathrm{t}$, $J=7.5 \mathrm{~Hz}), 7.05-6.96(3 \mathrm{H}, \mathrm{m}), 5.19(2 \mathrm{H}, \mathrm{s}) ;{ }^{13} \mathrm{C} \mathrm{NMR}\left(\mathrm{CDCb}_{3}, 75 \mathrm{MHz}\right) \delta 157.95$, 147.41, 144.51, 129.61, 127.53, 123.76, 121.53, 114.80, 68.74; LRMS (EI): $\mathrm{m} / z=230$ (23), 229 (93), 137 (15), 136 (100), 106 (42), 90 (28), 89 (25), 84 (14), 78 (45), 65 (14), 63 (11); HRMS (EI): $\mathrm{m} / z$ calcd. for $\left(\mathrm{C}_{13} \mathrm{H}_{11} \mathrm{NO}_{3}{ }^{+}\right)=229.0739$, found $=229.0734$.

(4-Methoxybenzyl)phenyl ether (2g) (Forrester, J.; Jones, R. V. H.; Newton, L.; Preston, P. N. Tetrahedron 2001, 57, 2871-2884.) 
Isolated as a white, crystalline solid in $80 \%$ yield: $\mathrm{R}=0.4\left(9: 1\right.$ hexanes:EtOAc); ${ }^{1} \mathrm{H}$ NMR (CDCb, $300 \mathrm{MHz}) \delta 7.40$ - $7.28(4 \mathrm{H}, \mathrm{m}), 7.03$ - $6.92(5 \mathrm{H}, \mathrm{m}), 5.01(2 \mathrm{H}, \mathrm{s}), 3.84$ $(3 \mathrm{H}, \mathrm{s}) ;{ }^{13} \mathrm{C} \mathrm{NMR}\left(\mathrm{CDCl}_{3}, 75 \mathrm{MHz}\right) \delta 159.30,158.71,129.42,129.20,129.07,120.85$, 114.86, 114.03, 69.88, 55.54.

2-Phenoxymethyl furan (2h) (Katritzky, A. R.; Abdel-Megeed, M. F.; Lhommet, G.; Ramsden, C. A. J. Chem. Soc. Perkin Trans. 1 1979, 426-429.)

Isolated as a clear oil in $90 \%$ yield: $\mathrm{R}_{f}=0.6$ (9:1 hexanes:EtOAc); ${ }^{1} \mathrm{H}$ NMR $\left(\mathrm{CDCl}_{3}, 300\right.$ MHz) $\delta 7.46(1 \mathrm{H}, \mathrm{dd}, J=2.0,1.0 \mathrm{~Hz}), 7.35-7.28(2 \mathrm{H}, \mathrm{m}), 7.04-6.96(3 \mathrm{H}, \mathrm{m}), 6.45(1 \mathrm{H}$, $\mathrm{dd}, J=3.5,0.5 \mathrm{~Hz}), 6.39(1 \mathrm{H}, \mathrm{dd}, J=3.5,2.0 \mathrm{~Hz}), 5.02(2 \mathrm{H}, \mathrm{s}) ;{ }^{13} \mathrm{C} \mathrm{NMR}\left(\mathrm{CDCl}_{3}, 75\right.$ MHz) $\delta 158.24,150.24,142.98,129.44,121.23,114.94,110.53,109.91,62.57$.

(2-Butynyl)phenyl ether (2i) (Larock, R. C.; Harrison, L. W. J. Amer. Chem. Soc. 1984, 106, 4218-4227.)

Isolated as a clear oil in $92 \%$ yield: $\mathrm{R}_{f}=0.7$ (9:1 hexanes:EtOAc); ${ }^{1} \mathrm{H}$ NMR $\left(\mathrm{CDCl}_{3}, 300\right.$ $\mathrm{MHz}) \delta 7.31(2 \mathrm{H}, \mathrm{t}, J=7.5 \mathrm{~Hz}), 7.02-6.94(3 \mathrm{H}, \mathrm{m}), 4.66(2 \mathrm{H}, \mathrm{q}, J=2.0 \mathrm{~Hz}), 1.88(3 \mathrm{H}$, $\mathrm{t}, J=2.0 \mathrm{~Hz}) ;{ }^{13} \mathrm{C} \mathrm{NMR}\left(\mathrm{CDCl}_{3}, 75 \mathrm{MHz}\right) \delta 157.69,129.37,121.19,114.83,83.77$, $74.26,56.56,4.11$.

\section{1, 8-diphenoxy-octa-3, 5-diyne (2j)}

Isolated as a beige, needle-like, crystalline solid in $61 \%$ yield: $\mathrm{R}=0.7$ (9:1 hexanes: EtOAc); mp $=133-135{ }^{\circ} \mathrm{C}$ (hexanes); ${ }^{1} \mathrm{H}$ NMR $\left(\mathrm{CDCl}_{3}, 300 \mathrm{MHz}\right) \delta 7.28(4 \mathrm{H}, \mathrm{t}, J=8.0$ $\mathrm{Hz}), 6.96(2 \mathrm{H}, \mathrm{t}, J=8.0 \mathrm{~Hz}), 6.89(4 \mathrm{H}, \mathrm{d}, J=8.0 \mathrm{~Hz}), 4.09(4 \mathrm{H}, \mathrm{t}, J=7.0 \mathrm{~Hz}), 2.76(4 \mathrm{H}$, $\mathrm{t}, J=7.0 \mathrm{~Hz}) ;{ }^{13} \mathrm{C} \mathrm{NMR}\left(\mathrm{CDCl}_{3}, 75 \mathrm{MHz}\right) \delta 158.17,129.50,121.18,114.70,74.06$, 66.81, 65.77, 20.73; IR (film) v 2936, 1597, 1499, 1470, 1390, 1304, 1246, 1176, 1082, 1041, 883, 812, 752, $689 \mathrm{~cm}^{-1}$; LRMS (EI): $m / z=291$ (18), 290 (68), 289 (15), 262 (19), 261 (16), 183 (41), 182 (17), 181 (23), 157 (15), 153 (16), 133 (52), 107 (34), 79 (31), 78 (15), 77 (100), 65 (19), 63 (17), 51 (29); HRMS (EI): $m / z$ calcd. for $\left(\mathrm{C}_{20} \mathrm{H}_{18} \mathrm{O}_{2}{ }^{+}\right)=$ 290.1307 , found $=290.1307$.

Phenyl-2,2,2-trifluoroethyl ether (2k) (Keegstra, M. A.; Peters, T. H. A.; Brandsma, L. Tetrahedron 1992, 48, 3633-3652.)

Isolated as a clear oil in $82 \%$ yield: $\mathrm{R}_{f}=0.7$ (9:1 hexanes:EtOAc); ${ }^{1} \mathrm{H}$ NMR $\left(\mathrm{CDCl}_{3}, 300\right.$ $\mathrm{MHz}) \delta 7.38$ - $7.30(2 \mathrm{H}, \mathrm{m}), 7.10-7.02(1 \mathrm{H}, \mathrm{m}), 6.99-6.93(2 \mathrm{H}, \mathrm{m}), 4.36(2 \mathrm{H}, \mathrm{q}, J=8.0$ $\mathrm{Hz}) ;{ }^{13} \mathrm{C} \mathrm{NMR}\left(\mathrm{CDCl}_{3}, 75 \mathrm{MHz}\right) \delta 157.30,129.73,122.54,114.99,66.08(\mathrm{q}, J=35.5$ $\mathrm{Hz}) ;{ }^{19} \mathrm{~F} \mathrm{NMR}\left(\mathrm{CDCl}_{3}, 282 \mathrm{MHz}\right) \delta-73.98(3 \mathrm{~F}, \mathrm{t}, J=8.0 \mathrm{~Hz})$. 
(3-Chloropropyl)phenyl ether (2l) (Foubelo, F.; Saleh, S. A.; Yus, M. J. Org. Chem. 2000, 65, 3478-3483.)

Isolated as a clear oil in $95 \%$ yield: $\mathrm{R}_{f}=0.7$ (9:1 hexanes:EtOAc); ${ }^{1} \mathrm{H}$ NMR $\left(\mathrm{CDCl}_{3}, 300\right.$ $\mathrm{MHz}) \delta 7.34-7.26(2 \mathrm{H}, \mathrm{m}), 7.00-6.90(3 \mathrm{H}, \mathrm{m}), 4.13(2 \mathrm{H}, \mathrm{t}, J=6.0 \mathrm{~Hz}), 3.77(2 \mathrm{H}, \mathrm{t}, J=$ $6.0 \mathrm{~Hz}), 2.26(2 \mathrm{H}$, quintet, $J=6.0 \mathrm{~Hz}) ;{ }^{13} \mathrm{C} \mathrm{NMR}\left(\mathrm{CDCl}_{3}, 75 \mathrm{MHz}\right) \delta 158.57,129.47$, $120.91,114.56,64.38,41.85,32.66$.

(3-Bromopropyl)phenyl ether (2m) (Reinholz, E.; Becker, A.; Hagenbruch, B.; Schaefer, S.; Schmitt, A. Synthesis 1990, 11, 1069-1071.)

Isolated as a clear oil in $93 \%$ yield: $\mathrm{R}_{f}=0.7$ (9:1 hexanes:EtOAc); ${ }^{1} \mathrm{H}$ NMR $\left(\mathrm{CDCl}_{3}, 300\right.$ $\mathrm{MHz}) \delta 7.36-7.31(2 \mathrm{H}, \mathrm{m}), 7.03-6.94(3 \mathrm{H}, \mathrm{m}), 4.14(2 \mathrm{H}, \mathrm{t}, J=6.0 \mathrm{~Hz}), 3.65(2 \mathrm{H}, \mathrm{t}, J=$ $6.0 \mathrm{~Hz}), 2.36(2 \mathrm{H}$, quintet, $J=6.0 \mathrm{~Hz}) ;{ }^{13} \mathrm{C} \mathrm{NMR}\left(\mathrm{CDCl}_{3}, 75 \mathrm{MHz}\right) \delta 158.79,129.66$, $121.06,114.63,65.27,32.54,30.29$.

Phenyl-p -tolyl ether (2n) (Haga, N.; Takayanagi, H. J. Org. Chem. 1996, 61, 735-745.) Isolated as a clear oil in $82 \%$ yield: $\mathrm{R}_{f}=0.60$ (5:1 hexanes:toluene); ${ }^{1} \mathrm{H}$ NMR $(300 \mathrm{MHz}$, $\left.\mathrm{CDCl}_{3}\right) \delta 7.50-7.41(2 \mathrm{H}, \mathrm{m}), 7.30-7.05(7 \mathrm{H}, \mathrm{m}), 2.48(3 \mathrm{H}, \mathrm{s}) ;{ }^{13} \mathrm{C}$ NMR $(100 \mathrm{MHz}$, $\left.\mathrm{CDCl}_{3}\right) \delta 158.00,154.90,133.03,130.40,129.81,123.37,119.30,118.51,20.86$.

4-Cyanodiphenyl ether (2o) (Gujadhur, R.; Venkataraman, D. Synth. Commun. 2001, 31, 2865-2880.)

Isolated as a thick, yellow oil in $77 \%$ yield: $\mathrm{R}_{f}=0.70$ (3:1 hexanes:EtOAc); ${ }^{1} \mathrm{H}$ NMR $\left(300 \mathrm{MHz}, \mathrm{CDCb}_{3}\right) \delta 7.60(2 \mathrm{H}, \mathrm{ddd}, J=9.5,5.0,2.5 \mathrm{~Hz}), 7.42(2 \mathrm{H}, \mathrm{t}, J=7.5 \mathrm{~Hz}), 7.23$ $(1 \mathrm{H}, \mathrm{tt}, J=7.0,1.0 \mathrm{~Hz}), 7.06(2 \mathrm{H}$, ddd, $J=7.5,3.5,1.0 \mathrm{~Hz}), 7.00(2 \mathrm{H}, \mathrm{ddd}, J=9.5,5.0$, $2.5 \mathrm{~Hz}) ;{ }^{13} \mathrm{C} \mathrm{NMR}\left(75 \mathrm{MHz}, \mathrm{CDCl}_{3}\right) \delta 161.84,154.99,134.30,130.41,125.32,120.58$, 119.02, 118.09, 106.00.

Allylphenyl ether (2p) (Annunziata, A.; Galli, C.; Marinelli, M.; Pau, T. Eur. J. Org. Chem. 2001, 7, 1323-1330.)

Isolated as a clear oil in $93 \%$ yield: $\mathrm{R}_{f}=0.8$ (9:1 hexanes:EtOAc); ${ }^{1} \mathrm{H}$ NMR $\left(\mathrm{CDCl}_{3}, 300\right.$ MHz) $\delta 7.38$ - $7.28(2 \mathrm{H}, \mathrm{m}), 7.00-6.91(3 \mathrm{H}, \mathrm{m}), 6.09$ (1H, ddt, $J=17.5,10.5,5.5 \mathrm{~Hz})$, $5.44(1 \mathrm{H}, \mathrm{dd}, J=17.5,1.5 \mathrm{~Hz}), 5.31(1 \mathrm{H}, \mathrm{dd}, J=10.5,1.5 \mathrm{~Hz}), 4.55(2 \mathrm{H}, \mathrm{dt}, J=5.5,1.5$ $\mathrm{Hz}) ;{ }^{13} \mathrm{C} \mathrm{NMR}\left(\mathrm{CDCl}_{3}, 75 \mathrm{MHz}\right) \delta 158.71,133.49,129.58,120.97,117.76,114.85$, 68.82 . 


\section{(E)-Pent-2-enylphenyl ether (2q)}

Isolated as a clear oil in $74 \%$ yield: $\mathrm{R}_{f}=0.4$ (3:1 hexanes:toluene); ${ }^{1} \mathrm{H}$ NMR $\left(\mathrm{CDCl}_{3}, 300\right.$ $\mathrm{MHz}) \delta 7.28(2 \mathrm{H}, \mathrm{d}, J=8.0 \mathrm{~Hz}), 6.98-6.90(3 \mathrm{H}, \mathrm{m}), 5.91(1 \mathrm{H}, \mathrm{dt}, J=15.5,6.0 \mathrm{~Hz})$, $5.72(1 \mathrm{H}, \mathrm{ddd}, J=15.5,6.0,1.0 \mathrm{~Hz}), 4.49(2 \mathrm{H}, \mathrm{dd}, J=6.0,1.0 \mathrm{~Hz}), 2.14(2 \mathrm{H}, \mathrm{ddq}, J=$ 7.5, 7.5, $1.0 \mathrm{~Hz}), 1.06(3 \mathrm{H}, \mathrm{t}, J=7.5 \mathrm{~Hz}) ;{ }^{13} \mathrm{C} \mathrm{NMR}\left(\mathrm{CDCl}_{3}, 75 \mathrm{MHz}\right) \delta 158.61,137.10$, 129.37, 123.84, 120.66, 114.74, 68.94, 25.72, 13.60; IR (film) v 2964, 1600, 1496, 1461, 1381, 1301, 1241, 1172, 1078, 1030, 1011, 968, 881, 753, $691 \mathrm{~cm}^{-1}$; LRMS (EI): $\mathrm{m} / z=$ 162 (8), 95 (12), 94 (100), 77 (8), 69 (16), 68 (19), 67 (8), 66 (6), 65 (9); HRMS (EI): $m / z$ calcd. for $\left(\mathrm{C}_{11} \mathrm{H}_{14} \mathrm{O}^{+}\right)=162.1045$, found $=162.1037$.

\section{(Z)-Pent-2-enylphenyl ether (2r)}

${ }^{1} \mathrm{H}$ NMR analysis of the starting material reveals a 1:20 ratio of E:Z geometric isomers which is carried through to the cross-coupled products. Isolated as a clear oil in $83 \%$ yield: $\mathrm{R}_{f}=0.6\left(3: 1\right.$ hexanes:toluene); ${ }^{1} \mathrm{H}$ NMR $\left(\mathrm{CDCl}_{3}, 300 \mathrm{MHz}\right) \delta 7.32-7.25(2 \mathrm{H}, \mathrm{m})$, 6.98 - $6.90(3 \mathrm{H}, \mathrm{m}), 5.70-5.65(2 \mathrm{H}, \mathrm{m}), 4.60(2 \mathrm{H}, \mathrm{d}, J=5.5 \mathrm{~Hz}), 2.23-2.15(2 \mathrm{H}, \mathrm{m})$, $1.05(3 \mathrm{H}, \mathrm{t}, J=7.5 \mathrm{~Hz}) ;{ }^{13} \mathrm{C} \mathrm{NMR}\left(\mathrm{CDCl}_{3}, 75 \mathrm{MHz}\right) \delta 158.60,135.96,129.39,124.17$, 120.71, 114.74, 63.99, 21.52, 14.49; IR (film) v 3028, 2965, 2874, 1600, 1586, 1496, 1300, 1242, 1172, 1078, 1031, 1011, 991, 881, 753, $691 \mathrm{~cm}^{-1}$; LRMS (EI): $\mathrm{m} / z=162$ (30), 95 (12), 94 (100), 77 (13), 69 (20), 68 (24), 67 (11), 66 (10), 65 (20), 53 (7), 51 (10).

Geranylphenyl ether (2s) (Nakamura, S.; Ishihara, K; Yamamoto, H. J. Amer. Chem. Soc. 2000, 122, 8131-8140.)

Isolated as a clear oil in $87 \%$ yield: $\mathrm{R}_{f}=0.6$ (4:1 hexanes:toluene); ${ }^{1} \mathrm{H}$ NMR $\left(\mathrm{CDCl}_{3}, 300\right.$ $\mathrm{MHz}) \delta 7.34-7.26(2 \mathrm{H}, \mathrm{m}), 7.00-6.91(3 \mathrm{H}, \mathrm{m}), 5.53(1 \mathrm{H}, \mathrm{tq}, J=3.5,1.5 \mathrm{~Hz}), 5.18-$ $5.00(1 \mathrm{H}, \mathrm{m}), 4.57(2 \mathrm{H}, \mathrm{d}, J=6.0 \mathrm{~Hz}), 2.23-2.08(4 \mathrm{H}, \mathrm{m}), 1.78(3 \mathrm{H}, \mathrm{s}), 1.73(3 \mathrm{H}, \mathrm{s})$, $1.66(3 \mathrm{H}, \mathrm{s}) ;{ }^{13} \mathrm{C} \mathrm{NMR}\left(\mathrm{CDCl}_{3}, 75 \mathrm{MHz}\right) \delta 158.74,140.91,131.70,129.34,123.83$, $120.55,119.64,114.71,64.95,39.86,26.67,26.03,18.07,17.02$.

(R)-2-Methyl-3-phenoxypropionic acid methyl ester (2t) (Toda, S.; Miyamoto, M.;

Kinoshita, H.; Inomata, K. Bull. Chem. Soc. Jpn. 1991, 64, 3600-3606.)

Isolated as a clear oil in $90 \%$ yield: $R_{f}=0.5\left(9: 1\right.$ hexanes:EtOAc); $[\alpha]_{\mathrm{D}}{ }^{22}=-13.2^{\circ}(\mathrm{c}=$ $3.1, \mathrm{CH}_{2} \mathrm{Cl}_{2}$ ) for $>99 \%$ ee (Chiralcel OD, $1 \%$ isopropanol/hexanes, $1.0 \mathrm{~mL} / \mathrm{min}$, retention times for $\mathrm{R}$ and $\mathrm{S}$ enantiomers are 8.26 and $9.98 \mathrm{~min}$ respectively) with determination of the absolute stereochemistry by comparison of the specific rotation with the literature value; ${ }^{1} \mathrm{H} \mathrm{NMR}\left(\mathrm{CDCl}_{3}, 300 \mathrm{MHz}\right) \delta 7.32$ - $7.25(2 \mathrm{H}, \mathrm{m}), 6.99$ - $6.87(3 \mathrm{H}, \mathrm{m}), 4.20(1 \mathrm{H}$, 
dd, $J=9.0,6.5 \mathrm{~Hz}$ ), $4.00(1 \mathrm{H}, \mathrm{dd}, J=9.0,6.5 \mathrm{~Hz}), 3.73(3 \mathrm{H}, \mathrm{s}), 2.97(1 \mathrm{H}$, sextet, $J=7.0$ $\mathrm{Hz}), 1.32(3 \mathrm{H}, \mathrm{d}, J=7.0 \mathrm{~Hz}) ;{ }^{13} \mathrm{C} \mathrm{NMR}\left(\mathrm{CDCl}_{3}, 75 \mathrm{MHz}\right) \delta 174.44,158.49,129.39$, 120.96, 114.68, 69.65, 52.11, 40.07, 14.38.

\section{Methyl-(2-phenoxyethyl)carbamic acid tert-butyl ester (2u)}

Isolated as a clear oil in $93 \%$ yield: $\mathrm{R}_{\mathrm{f}}=0.6\left(9: 1\right.$ hexanes: EtOAc); ${ }^{1} \mathrm{H} \mathrm{NMR}\left(\mathrm{CDCl}_{3}\right.$, $300 \mathrm{MHz}) \delta 7.28(2 \mathrm{H}, \mathrm{t}, J=7.5 \mathrm{~Hz}), 6.94(1 \mathrm{H}, \mathrm{t}, J=7.5 \mathrm{~Hz}), 6.89(2 \mathrm{H}, \mathrm{d}, J=7.5 \mathrm{~Hz})$, $4.09(2 \mathrm{H}, \mathrm{t}, J=5.5 \mathrm{~Hz}), 3.60(2 \mathrm{H}, \mathrm{t}, J=5.5 \mathrm{~Hz}), 2.99(3 \mathrm{H}, \mathrm{s}), 1.47(9 \mathrm{H}, \mathrm{s}) ;{ }^{13} \mathrm{C} \mathrm{NMR}$ $\left(\mathrm{CDCl}_{3}, 75 \mathrm{MHz}\right) \delta 158.47,155.53,129.41,120.81,114.41,79.69,66.49$ (broad), 48.57, 36.10 (broad), 28.75; IR (film) v 2976, 1697, 1600, 1497, 1391, 1366, 1303, 1246, 1157, 1046, 882, $754 \mathrm{~cm}^{-1}$; LRMS (EI): $\mathrm{m} / \mathrm{z}=251$ (5), 196 (11), 179 (17), 178 (90), 158 (31), 120 (19), 108 (14), 107 (11), 103 (10), 102 (100), 94 (15), 77 (29), 58 (11), 57 (59); HRMS (EI): $\mathrm{m} / z$ calcd. for $\left(\mathrm{C}_{14} \mathrm{H}_{21} \mathrm{NO}_{3}+\mathrm{H}^{+}\right)=252.1600$, found $=252.1609$.

\section{(3-Phenoxypropyl)carbamic acid benzyl ester (2v)}

Isolated as a clear oil in $85 \%$ yield: $\mathrm{R}_{\mathrm{f}}=0.4$ (3:1 hexanes: EtOAc); ${ }^{1} \mathrm{H}$ NMR $\left(\mathrm{CDCb}_{3}\right.$, $300 \mathrm{MHz}) \delta 7.38-7.25(7 \mathrm{H}, \mathrm{m}), 6.98-6.86(3 \mathrm{H}, \mathrm{m}), 5.12(2 \mathrm{H}, \mathrm{s}), 5.08(1 \mathrm{H}, \mathrm{s}$, broad $)$, $4.03(2 \mathrm{H}, \mathrm{t}, J=6.0 \mathrm{~Hz}), 3.42(2 \mathrm{H}, \mathrm{q}, J=6.0 \mathrm{~Hz}), 2.02(2 \mathrm{H}$, quintet, $J=6.0 \mathrm{~Hz}) ;{ }^{13} \mathrm{C}$ NMR $\left(\mathrm{CDCl}_{3}, 75 \mathrm{MHz}\right) \delta 158.51,156.32,136.53,129.44,128.47,128.04,120.87$, 114.48, 66.83, 65.85, 38.96, 29.72 (one signal absent); IR (film) $\vee 3336,3064,3033$, 2949, 2878, 1702, 1600, 1587, 1530, 1498, 1471, 1455, 1243, 1173, 1142, 1080, 1041, 754, $693 \mathrm{~cm}^{-1}$; LRMS (EI): $\mathrm{m} / z=193$ (12), 192 (84), 177 (7), 121 (5), 108 (9), 107 (9), 94 (20), 92 (10), 91 (100), 84 (6), 79 (10), 77 (15), 65 (17), 56 (10), 51 (8); HRMS (EI): $\mathrm{m} / z$ calcd. for $\left(\mathrm{C}_{17} \mathrm{H}_{19} \mathrm{NO}_{3}{ }^{+}\right)=285.1365$, found $=285.1374$.

\section{Methyl-(2-phenoxyethyl)phenyl amine (2w)}

Isolated as a clear oil in $30 \%$ yield: $\mathrm{R}_{f}=0.5$ (9:1 hexanes: EtOAc); ${ }^{1} \mathrm{H}$ NMR $\left(\mathrm{CDCb}_{3}\right.$, $300 \mathrm{MHz}) \delta 7.34-7.25(4 \mathrm{H}, \mathrm{m}), 7.01-6.90(3 \mathrm{H}, \mathrm{m}), 6.83-6.73(3 \mathrm{H}, \mathrm{m}), 4.17(2 \mathrm{H}, \mathrm{t}, J=$ $6.0 \mathrm{~Hz}), 3.80(2 \mathrm{H}, \mathrm{t}, J=6.0 \mathrm{~Hz}), 3.09(3 \mathrm{H}, \mathrm{s}) ;{ }^{13} \mathrm{C} \mathrm{NMR}\left(\mathrm{CDCl}_{3}, 75 \mathrm{MHz}\right) \delta 158.83$, 149.07, 129.64, 129.45, 121.02, 116.63, 114.60, 112.25, 65.21, 52.05, 39.30; IR (film) $v$ 3027, 2923, 1599, 1497, 1368, 1301, 1245, 1172, 1077, 1034, 992, 884, 749, $691 \mathrm{~cm}^{-1}$; LRMS (EI): $m / z=228$ (9), 227 (48), 212 (11), 121 (14), 120 (100), 105 (8), 104 (8), 91 (5), 84 (6), 77 (19), 51 (7); HRMS (EI): $m / z$ calcd. for $\left(\mathrm{C}_{15} \mathrm{H}_{17} \mathrm{NO}^{+}\right)=227.1310$, found $=$ 227.1307 . 
Isopropylphenyl ether (2x) (Keegstra, M. A.; Peters, T. H. A.; Brandsma, L. Tetrahedron 1992, 48, 3633-3652.)

Isolated as a clear oil in $71 \%$ yield: $\mathrm{R}_{f}=0.8$ (9:1 hexanes:EtOAc); ${ }^{1} \mathrm{H}$ NMR $\left(\mathrm{CDCl}_{3}, 300\right.$ MHz) $\delta 7.30$ - $7.24(2 \mathrm{H}, \mathrm{m}), 6.98-6.88(3 \mathrm{H}, \mathrm{m}), 4.57(1 \mathrm{H}$, septet, $J=6.0 \mathrm{~Hz}), 1.37(6 \mathrm{H}$, $\mathrm{d}, J=6.0 \mathrm{~Hz}) ;{ }^{13} \mathrm{C} \mathrm{NMR}\left(\mathrm{CDCl}_{3}, 75 \mathrm{MHz}\right) \delta 157.77,129.43,120.49,115.94,69.94$, 22.46 .

\section{(1-Methylallyl)phenyl ether (2y)}

Isolated as a clear oil in $69 \%$ yield: $\mathrm{R}_{f}=0.8$ (9:1 hexanes:EtOAc); ${ }^{1} \mathrm{H}$ NMR $\left(\mathrm{CDCl}_{3}, 300\right.$ MHz) $\delta 7.38$ - $7.30(2 \mathrm{H}, \mathrm{m}), 6.96-6.89$ (3H, m), 5.93 (1H, ddd, $J=17.5,10.5,6.0 \mathrm{~Hz})$, $5.28(1 \mathrm{H}, \mathrm{dd}, J=17.5,1.0 \mathrm{~Hz}), 5.18(1 \mathrm{H}, \mathrm{dd}, J=10.5,1.0 \mathrm{~Hz}), 4.82(1 \mathrm{H}, \mathrm{ddq}, J=6.0$, 6.0, $1.0 \mathrm{~Hz}), 1.46(3 \mathrm{H}, \mathrm{d}, J=6.0 \mathrm{~Hz}) ;{ }^{13} \mathrm{C} \mathrm{NMR}\left(\mathrm{CDCb}_{3}, 75 \mathrm{MHz}\right) \delta 157.88,139.19$, 129.31, 120.73, 116.07, 115.57, 74.72, 21.67; IR (film) v 3040, 2981, 1585, 1488, 1287, $1239,1164,1072,1024,990,928,866,797,750 \mathrm{~cm}^{-1}$.

\section{Benzhydrylphenyl ether (2z)}

Isolated as a clear liquid in $75 \%$ yield: $\mathrm{R}_{f}=0.6$ (9:1 hexanes: EtOAc); ${ }^{1} \mathrm{H} \mathrm{NMR}\left(\mathrm{CDCl}_{3}\right.$, $300 \mathrm{MHz}) \delta 7.54$ - $7.26(12 \mathrm{H}, \mathrm{m}), 7.07$ - $6.95(3 \mathrm{H}, \mathrm{m}), 6.30(1 \mathrm{H}, \mathrm{s}) ;{ }^{13} \mathrm{C} \mathrm{NMR}\left(\mathrm{CDCl}_{3}\right.$, $75 \mathrm{MHz}) \delta 158.25,141.43,129.53,128.77,127.89,127.06,121.13,116.24,81.80$; IR (film) v 3062, 3029, 1597, 1586, 1493, 1454, 1306, 1235, 1172, 1078, 1029, 916, 880, 817, 753, 699, $655 \mathrm{~cm}^{-1}$; LRMS (EI): $\mathrm{m} / z=262$ (43), 260 (10), 183 (25), 168 (24), 167 (100), 166 (17), 165 (39), 152 (20), 65 (12); HRMS (EI): m/z calcd. for $\left(\mathrm{C}_{19} \mathrm{H}_{16} \mathrm{O}^{+}\right)=$ 260.1201 , found $=260.1196$.

Cyclohexylphenyl ether (2aa) (Tateiwa, J.; Nishimura, T.; Horiuchi, H.; Uemura, S. J. Chem. Soc. Perkin Trans.1 1994, 23, 3367-3372.)

Isolated as a clear oil in $67 \%$ yield: $\mathrm{R}_{f}=0.8$ (9:1 hexanes:EtOAc); ${ }^{1} \mathrm{H}$ NMR $\left(\mathrm{CDCl}_{3}, 300\right.$ MHz) $\delta 7.40$ - $7.25(2 \mathrm{H}, \mathrm{m}), 6.98$ - $6.90(3 \mathrm{H}, \mathrm{m}), 4.27(1 \mathrm{H}, \mathrm{m}), 2.10$ - $1.98(2 \mathrm{H}, \mathrm{m}), 1.91$ - $1.80(2 \mathrm{H}, \mathrm{m}), 1.68$ - $1.50(3 \mathrm{H}, \mathrm{m}), 1.48-1.28(3 \mathrm{H}, \mathrm{m}) ;{ }^{13} \mathrm{C} \mathrm{NMR}\left(\mathrm{CDCl}_{3}, 75 \mathrm{MHz}\right) \delta$ 157.72, 129.38, 120.47, 116.09, 75.50, 32.20, 26.02, 24.18.

\section{$O$-Phenyl stigmasterol (2bb)}

Isolated as a clear crystal in $48 \%$ yield: $R_{f}=0.9\left(9: 1\right.$ hexanes: EtOAc); $[\alpha]_{\mathrm{D}}{ }^{22}=-40.7^{\circ}$ $\left(\mathrm{c}=1.4, \mathrm{CH}_{2} \mathrm{Cl}_{2}\right) ;{ }^{1} \mathrm{H} \mathrm{NMR}\left(\mathrm{CDCl}_{3}, 300 \mathrm{MHz}\right) \delta 7.29-7.23(2 \mathrm{H}, \mathrm{m}), 6.94-6.88(3 \mathrm{H}$, m), $5.40(1 \mathrm{H}, \mathrm{d}, J=5.0 \mathrm{~Hz}), 5.17(1 \mathrm{H}, \mathrm{dd}, J=15.0,8.5 \mathrm{~Hz}), 5.02(1 \mathrm{H}, \mathrm{dd}, J=15.0,8.5$ $\mathrm{Hz}), 4.18$ - $4.07(1 \mathrm{H}, \mathrm{m}), 2.54-2.36(2 \mathrm{H}, \mathrm{m}), 2.11$ - $1.90(4 \mathrm{H}, \mathrm{m}), 1.76-1.66(2 \mathrm{H}, \mathrm{m})$, 
1.65 - $1.34(8 \mathrm{H}, \mathrm{m}), 1.33$ - $1.12(6 \mathrm{H}, \mathrm{m}), 1.10-1.02(9 \mathrm{H}, \mathrm{m}), 0.90-0.80(9 \mathrm{H}, \mathrm{m}), 0.73$ $(3 \mathrm{H}, \mathrm{s}) ;{ }^{13} \mathrm{C} \mathrm{NMR}\left(\mathrm{CDCl}_{3}, 75 \mathrm{MHz}\right) \delta 157.62,140.33,138.28,129.45,129.30,122.29$, $120.62,115.98,77.20,57.15,56.25,51.54,50.54,42.56,40.83,40.02,39.06,37.56$, $37.21,32.31,32.27,29.30,28.65,25.81,24.76,21.64,21.50,21.48,19.86,19.41,12.70$, 12.50; IR (film) v 3037, 2950, 2851, 2361, 1583, 1486, 1379, 1366, 1290, 1230, 1152, 1088, 1037, 1021, 972, 866, 839, 806, 758, $693 \mathrm{~cm}^{-1}$; LRMS (EI): $\mathrm{m} / z=488$ (1), 397 (18), 396 (27), 395 (64), 311 (10), 255 (28), 161 (14), 159 (15), 149 (13), 147 (17), 145 (13), 139 (41), 135 (12), 133 (14), 121 (19), 119 (11), 109 (15), 107 (16), 105 (20), 97 (23), 95 (24), 94 (17), 93 (22), 91 (18), 84 (14), 83 (100), 81 (31), 79 (18), 77 (10), 71 (19), 69 (47), 67 (16), 57 (28), 55 (42); HRMS (EI): m/z calcd. for $\left(\mathrm{C}_{35} \mathrm{H}_{52} \mathrm{O}^{+}\right)=$ 488.4018 , found $=488.4027$. 


\section{Products of Experiments Summarized in Table 2}

\section{2-(4-Methoxyphenoxymethyl) furan (3a)}

Isolated as a clear, crystalline solid in quantitative yield: $\mathrm{R}_{\mathrm{f}}=0.4$ (9:1 hexanes: EtOAc); ${ }^{1} \mathrm{H}$ NMR $\left(\mathrm{CDCl}_{3}, 300 \mathrm{MHz}\right) \delta 7.44(1 \mathrm{H}, \mathrm{dd}, J=2.0,1.0 \mathrm{~Hz}), 6.93(2 \mathrm{H}, \mathrm{d}, J=9.0 \mathrm{~Hz})$, $6.84(2 \mathrm{H}, \mathrm{d}, J=9.0 \mathrm{~Hz}), 6.41(1 \mathrm{H}, \mathrm{d}, J=3.0 \mathrm{~Hz}), 6.38(1 \mathrm{H}, \mathrm{dd}, J=3.0,2.0 \mathrm{~Hz}), 4.95$ $(2 \mathrm{H}, \mathrm{s}), 3.78(3 \mathrm{H}, \mathrm{s}) ;{ }^{13} \mathrm{C} \mathrm{NMR}\left(\mathrm{CDCl}_{3}, 75 \mathrm{MHz}\right) \delta 154.11,152.37,150.47,142.92$, 116.21, 114.62, 110.48, 109.80, 63.53, 55.89; IR (film) v 2933, 2834, 1592, 1508, 1464, 1371, 1232, 1150, 1108, 1037, 1010, 923, 825, 780, $744 \mathrm{~cm}^{-1}$; LRMS (EI): $m / z=204$ (32), 123 (18), 95 (10), 82 (8), 81 (100), 53 (18), 52 (6); HRMS (EI): m/z calcd. for $\left(\mathrm{C}_{12} \mathrm{H}_{12} \mathrm{O}_{3}{ }^{+}\right)=204.0786$, found $=204.0779$.

\section{2-(3-Chlorophenoxymethyl) furan (3b)}

Isolated as a clear oil in $71 \%$ yield: $\mathrm{R}_{f}=0.5$ (3:1 hexanes: toluene); ${ }^{1} \mathrm{H} \mathrm{NMR}\left(\mathrm{CDCl}_{3}\right.$, $300 \mathrm{MHz}) \delta 7.46(1 \mathrm{H}, \mathrm{dd}, J=2.0,1.0 \mathrm{~Hz}), 7.21(1 \mathrm{H}, \mathrm{t}, J=8.0 \mathrm{~Hz}), 7.02-6.96(2 \mathrm{H}, \mathrm{m})$, $6.88(1 \mathrm{H}$, ddd, $J=8.0,2.5,1.0 \mathrm{~Hz}), 6.44(1 \mathrm{H}, \mathrm{d}, J=3.0 \mathrm{~Hz}), 6.40(1 \mathrm{H}, \mathrm{dd}, J=3.0,2.0$ $\mathrm{Hz}), 4.99(2 \mathrm{H}, \mathrm{s}) ;{ }^{13} \mathrm{C} \mathrm{NMR}\left(\mathrm{CDCl}_{3}, 75 \mathrm{MHz}\right) \delta 158.89,149.59,143.16,134.78,130.19$, 121.39, 115.36, 113.40, 110.59, 110.27, 62.73; IR (film) v 3123, 3070, 2933, 2874, 1594, 1477, 1371, 1284, 1247, 1151, 1099, 1073, 1011, 924, 898, 768, 744, $680 \mathrm{~cm}^{-1}$; LRMS (EI): $m / z=210$ (6), 208 (8), 143 (5), 141 (16), 128 (11), 113 (7), 111 (13), 101 (6), 99 (16), 83 (7), 82 (11), 81 (100), 75 (10), 73 (8), 64 (6), 63 (12), 53 (30), 52 (6), 51 (9); HRMS (EI): $m / z$ calcd. for $\left(\mathrm{C}_{11} \mathrm{H}_{9} \mathrm{ClO}_{2}{ }^{+}\right)=208.0291$, found $=208.0293$.

\section{2-(Thiophen-3-yloxymethyl) furan (3c)}

Isolated as a yellow oil in $76 \%$ yield: $\mathrm{R}_{f}=0.5$ (3:1 hexanes: toluene); ${ }^{1} \mathrm{H}$ NMR ( $\mathrm{CDCl}_{3}$, $300 \mathrm{MHz}) \delta 7.46(1 \mathrm{H}, \mathrm{dd}, J=2.0,1.0 \mathrm{~Hz}), 7.18(1 \mathrm{H}, \mathrm{dd}, J=5.0,3.0 \mathrm{~Hz}), 6.80(1 \mathrm{H}$, ddd, $J=5.0,1.5,1.0 \mathrm{~Hz}), 6.45(1 \mathrm{H}, \mathrm{d}, J=3.5 \mathrm{~Hz}), 6.41-6.37(2 \mathrm{H}, \mathrm{m}), 4.97(2 \mathrm{H}, \mathrm{s}) ;{ }^{13} \mathrm{C} \mathrm{NMR}$ $\left(\mathrm{CDCl}_{3}, 75 \mathrm{MHz}\right) \delta 156.89,150.02,143.11,124.74,119.69,110.57,110.24,98.33$, 64.49; IR (film) v 3117, 2927, 1542, 1503, 1422, 1392, 1359, 1235, 1174, 1150, 1072, 1008, 953, 923, 874, 817, 752, $626 \mathrm{~cm}^{-1}$; LRMS (EI): $m / z=180$ (23), 82 (8), 81 (100), 53 (18); HRMS (EI): $m / z$ calcd. for $\left(\mathrm{C}_{12} \mathrm{H}_{12} \mathrm{O}_{3}{ }^{+}\right)=180.0245$, found $=180.0245$.

\section{2-Styryloxymethyl furan (3d)}

Isolated as a clear oil in $61 \%$ yield: $\mathrm{R}_{f}=0.6$ (9:1 hexanes: EtOAc); ${ }^{1} \mathrm{H} \mathrm{NMR}\left(\mathrm{CDCl}_{3}\right.$, $300 \mathrm{MHz}) \delta 7.46(1 \mathrm{H}, \mathrm{dd}, J=2.0,1.0 \mathrm{~Hz}), 7.29-7.23(4 \mathrm{H}, \mathrm{m}), 7.18-7.06(1 \mathrm{H}, \mathrm{m}), 7.05$ $(1 \mathrm{H}, \mathrm{d}, J=13.0 \mathrm{~Hz}), 6.44(1 \mathrm{H}, \mathrm{d}, J=3.0 \mathrm{~Hz}), 6.39(1 \mathrm{H}, \mathrm{dd}, J=3.0,2.0 \mathrm{~Hz}), 5.99(1 \mathrm{H}, \mathrm{d}$, $J=13.0 \mathrm{~Hz}), 4.85(2 \mathrm{H}, \mathrm{s}) ;{ }^{13} \mathrm{C} \mathrm{NMR}\left(\mathrm{CDCl}_{3}, 75 \mathrm{MHz}\right) \delta 150.02,146.99,143.22,136.00$, 
128.57, 125.86, 125.22, 110.55, 110.15, 107.39, 64.28; IR (film) v 3024, 2924, 1733, 1654, 1637, 1598, 1501, 1448, 1371, 1330, 1276, 1227, 1153, 1072, 1016, 993, 921, 884, 816, 748, $694 \mathrm{~cm}^{-1}$; LRMS (EI): m/z = 200 (3), 170 (5), 135 (17), 120 (8), 107 (46), 105 (39), 91 (17), 90 (9), 89 (11), 82 (18), 81 (100), 79 (42), 77 (37), 63 (8), 53 (33), 52 (9), $51(20)$.

\section{2-Hex-1-enyloxymethyl furan (3e)}

Isolated as a clear oil in $55 \%$ yield: $\mathrm{R}_{f}=0.7$ (9:1 hexanes: EtOAc); ${ }^{1} \mathrm{H} \mathrm{NMR}\left(\mathrm{CDCl}_{3}\right.$, $300 \mathrm{MHz}) \delta 7.41(1 \mathrm{H}, \mathrm{t}, J=1.5 \mathrm{~Hz}), 6.35(2 \mathrm{H}, \mathrm{d}, J=1.5 \mathrm{~Hz}), 6.28(1 \mathrm{H}, \mathrm{dt}, J=12.5,1.0$ $\mathrm{Hz}), 4.91(1 \mathrm{H}, \mathrm{dt}, J=12.5,7.5 \mathrm{~Hz}), 4.64(2 \mathrm{H}, \mathrm{s}), 1.98-1.88(2 \mathrm{H}, \mathrm{m}), 1.38-1.26(4 \mathrm{H}$, $\mathrm{m}), 0.94-0.86(3 \mathrm{H}, \mathrm{m}) ;{ }^{13} \mathrm{C} \mathrm{NMR}\left(\mathrm{CDCl}_{3}, 75 \mathrm{MHz}\right) \delta 150.64,145.13,142.89,110.41$, 109.58, 105.76, 63.51, 33.04, 27.68, 22.38, 14.30; IR (film) v 2958, 2926, 2858, 1672, 1653, 1503, 1456, 1369, 1290, 1226, 1157, 1126, 1080, 1016, 933, 885, 813, $738 \mathrm{~cm}^{-1}$; LRMS (EI): $m / z=180$ (7), 149 (9), 105 (11), 97 (7), 85 (10), 83 (6), 82 (8), 81 (100), 57 (13), 53 (9); HRMS (EI): $m / z$ calcd. for $\left(\mathrm{C}_{11} \mathrm{H}_{16} \mathrm{O}_{2}{ }^{+}\right)=180.1150$, found $=180.1154$. 

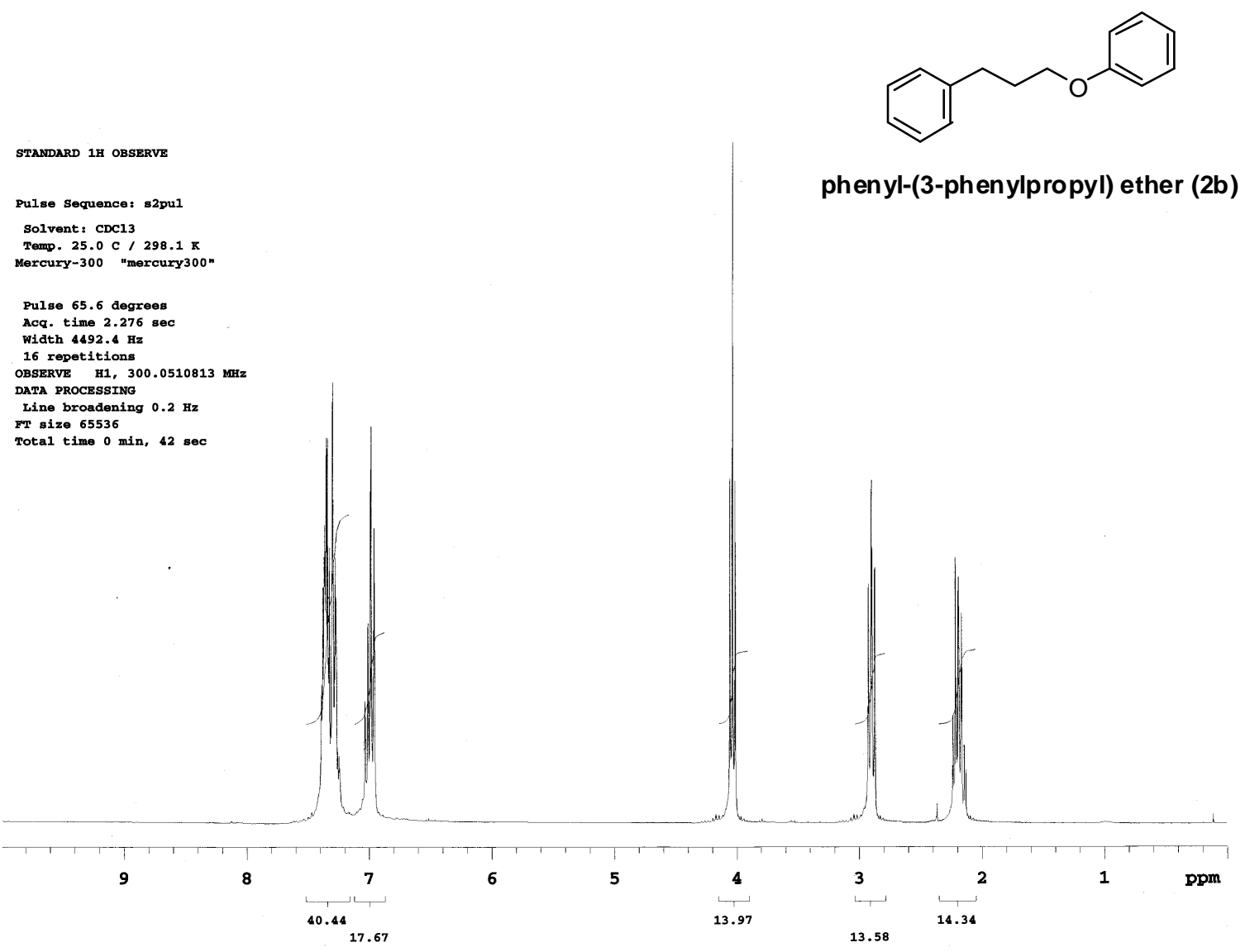

13C OBSERVE

Pulse Sequence: s2pul

Solvent: CDC13

Temp, 25.0 C / $298.1 \mathrm{~K}$

Mercury-300 "mercury300"

Pulse 45.0 degrees

Acq. time 1.000 sec

wiath $18797.0 \mathrm{~Hz}$

OBSERVE C13, 75.4479051 MHz

DECOUPLE H1, $300.0525807 \mathrm{MHZ}$

Power 39 dis

continuous1y on

waLTZ-16 modulated

DATA PROCESSTMG

Line broadening $1.0 \mathrm{~Hz}$

FT size 131072

Total time $54 \mathrm{~min}, 57 \mathrm{sec}$

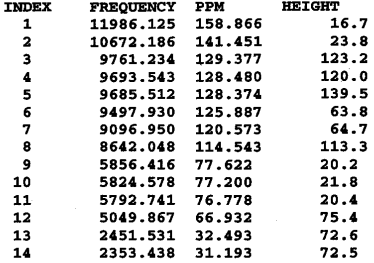

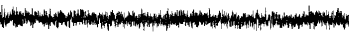

200

180

160

140
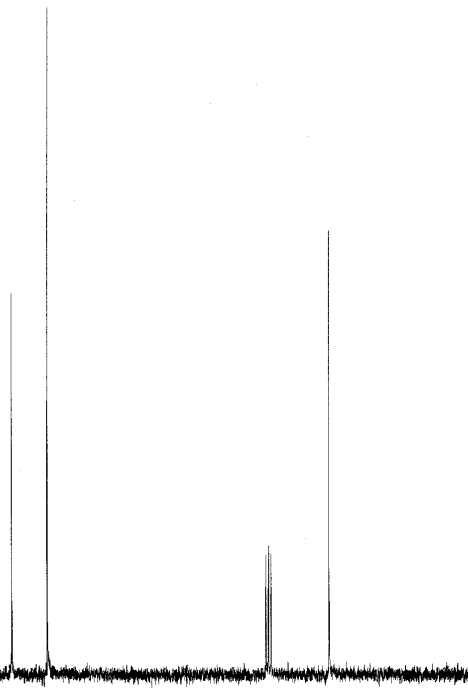


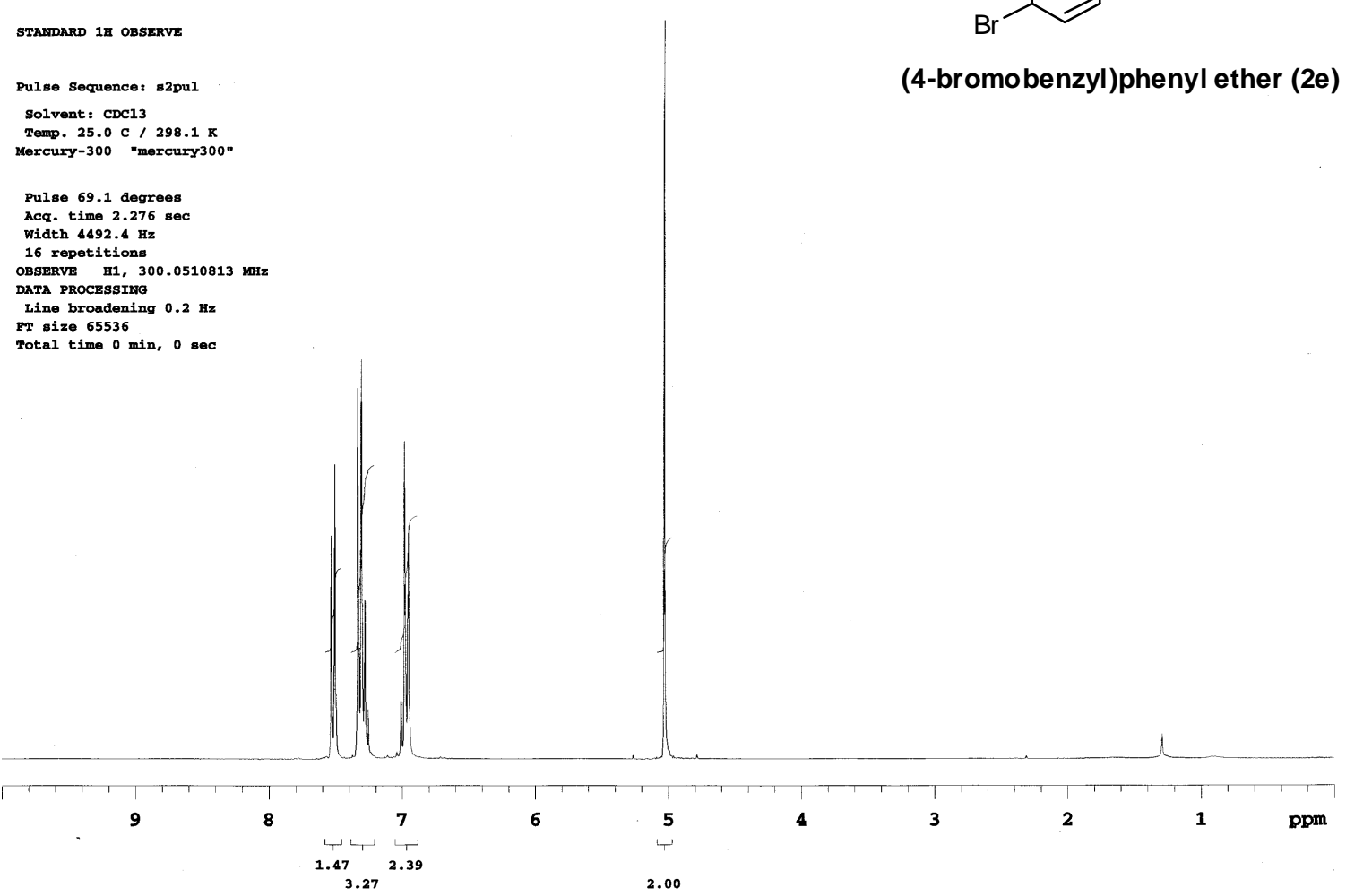

13C OBSERVE

Pulse Sequence: s2pu1

Solvent: $\mathrm{CDC13}$

Temp. $25.0 \mathrm{C} / 298.1 \mathrm{~K}$

Pulse 48.6 degrees

Acq. time 1.000 sec

wiath $18797.0 \mathrm{~Hz}$

684 repetitions

OBSERVE C13, 75.4477451 MHE

DECOUPLE H1, 300.0525807 MHZ

Power $40 \mathrm{~dB}$

continuously on

WALTZ-16 modulated

Line broaning 1.0

ET

Total time $1 \mathrm{hr}, 16 \mathrm{~min}, 22 \mathrm{sec}$

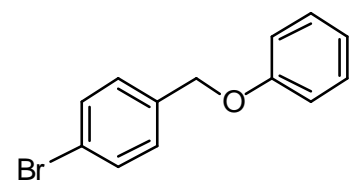

(4-bromobenzyl)phenyl ether (2e)

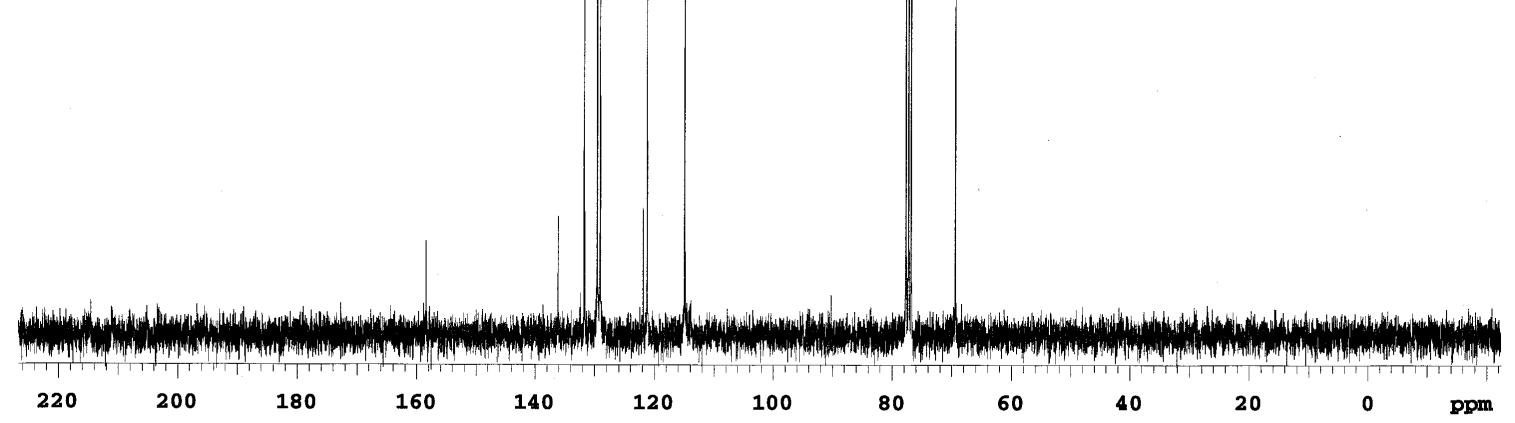




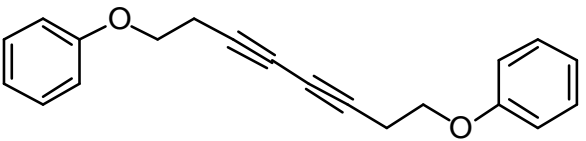

STANDARD 1H OBSERVE

Pulse Sequence: s2pul

Solvent: $\mathrm{CDC}_{13}$

Temp. $25.0 \mathrm{C} / 298.1 \mathrm{~K}$

Pulse 65.6 degrees

Acq. time $2.276 \mathrm{~s}$

wiath $4492.4 \mathrm{~Hz}$

OBSERVE H1, 300.0510813 MHZ

DATA PROCESSING

Line broadening

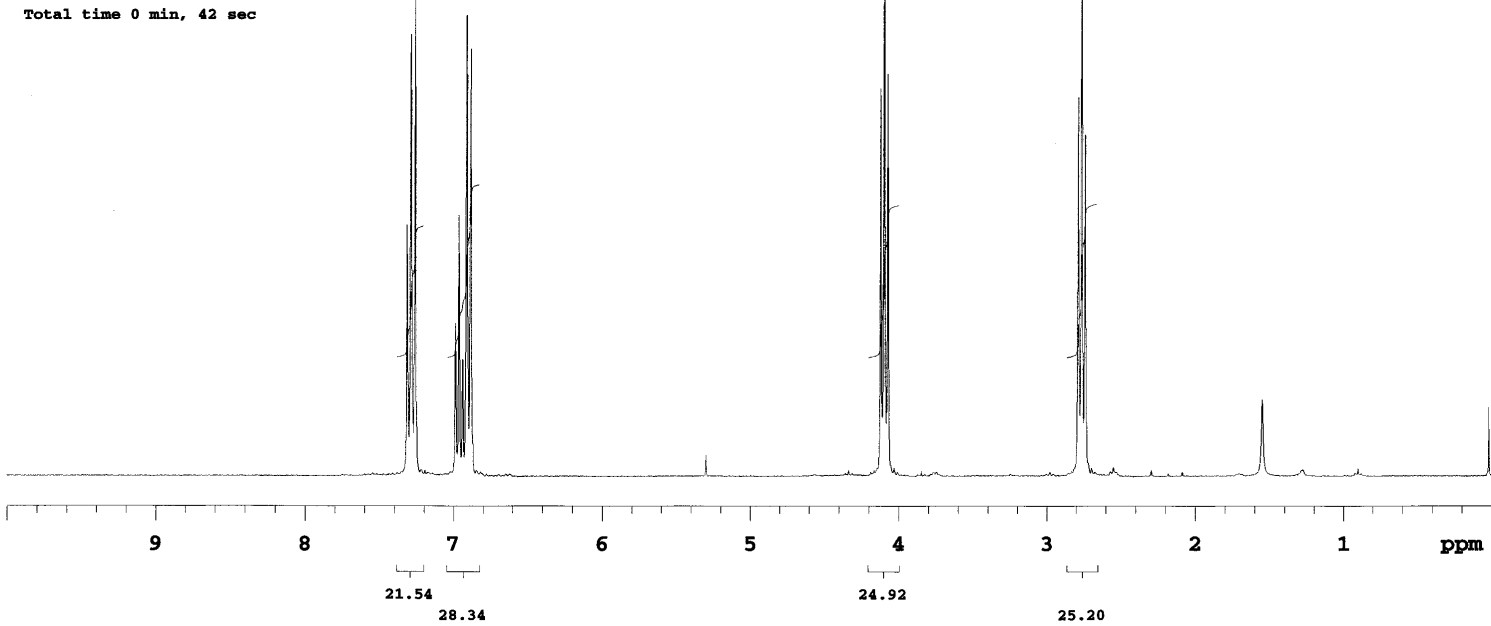

13C OBSERVE

Pulse Sequence: s2pul

Solvent: $\mathrm{CDC}_{13}$

Temp. $25.0 \mathrm{C} / 298.1 \mathrm{~K}$

Mercury-300 "mercury300"

Pulse 45.0 degrees

Acq. time $1.000 \mathrm{~s}$

960 repetitions

OBSERVE C13， 75.4478959 ME

DECOUPLE H1, $300.0525807 \mathrm{MHz}$

Power 39 dB

continuously on

WALTZ-16 modulat

DATA PROCESSING

Line broadening $1.0 \mathrm{H}$

Total time $54 \mathrm{~min}, 57 \mathrm{sec}$

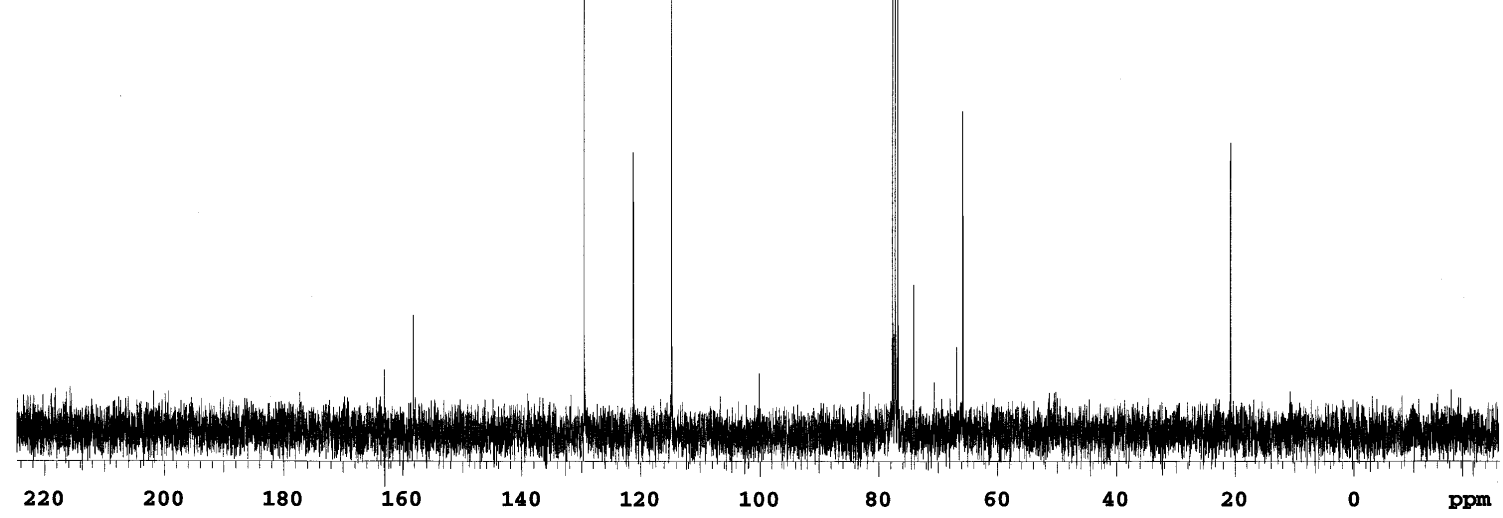




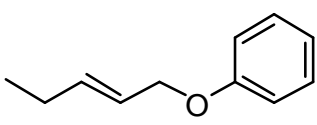

STANDARD 1H OBSERVE

Pu1se Sequence: s2pu]

Solvent: CDC13

Temp. $25.0 \mathrm{C} / 298.1 \mathrm{~K}$

"mercury300"

Pulse 69.1 degrees

Acq. time $2.276 \mathrm{sec}$

width $4492.4 \mathrm{~Hz}$

OBSERVE H1, 300.0510813 MHz

OBSERVE H1, 300

Line broadening $0.2 \mathrm{~Hz}$

Line broadening

Total time $0 \mathrm{~min}, 42 \mathrm{sec}$

\section{(E)-pent-2-enylphenyl ether (2q)}

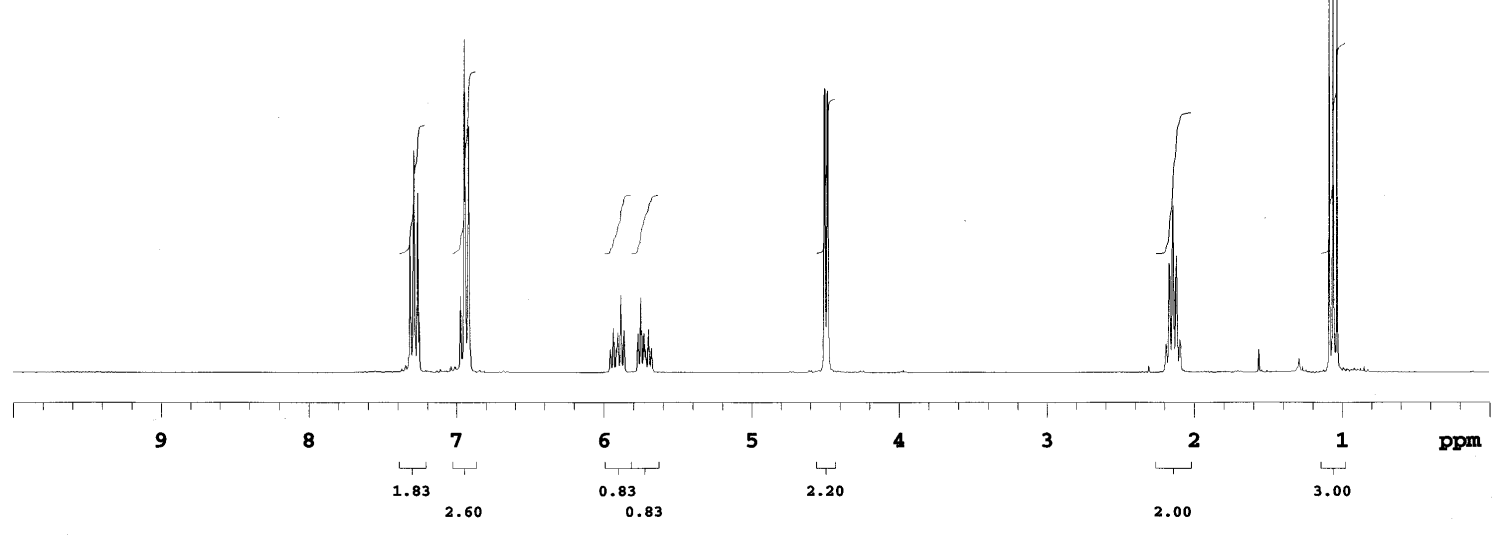

13C OBSERVE

Pu1se Sequence: s2pul

Solvent: $\mathrm{CDC} 13$

Temp. $25.0 \mathrm{C} / 298.1 \mathrm{~K}$

(1)

Relax. delay 0.500 sec

Pulse 48.6 degrees

Acq. time $1.000 \mathrm{sec}$

Width $18797.0 \mathrm{~Hz}$

208 repetitions

OBSERVE C13， $75.4478982 \mathrm{MHz}$

Din

Power 40 das

WALTZ-16 molulated

Dapa processivg

Line broadening 1.0

FT size 131072

Total time $1 \mathrm{hr}, 25 \mathrm{~min}, 17 \mathrm{sec}$

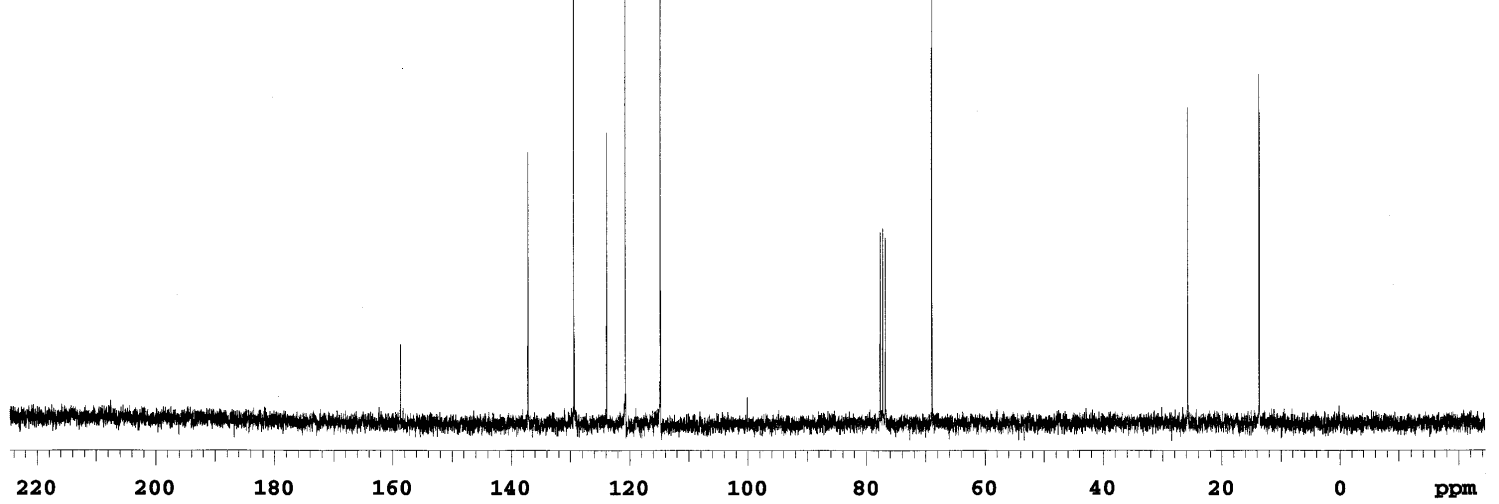




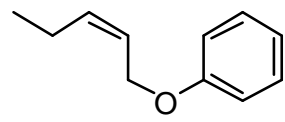

STANDARD 1H OBSERVE

(Z)-pent-2-enylphenyl ether (2r)

Pulse Sequence: s2pul

Solvent: $\mathrm{CDC1}_{3}$

Mercury-300 "mercury 300

Pulse 69.1 degrees

Acq. time 2.276 sec

16 th 4492.4 inz

16 repetitions

OBSERVE H1, 300

Line broadening 0.2

TT size 65536

Total time $0 \mathrm{~min}, 42 \mathrm{sec}$
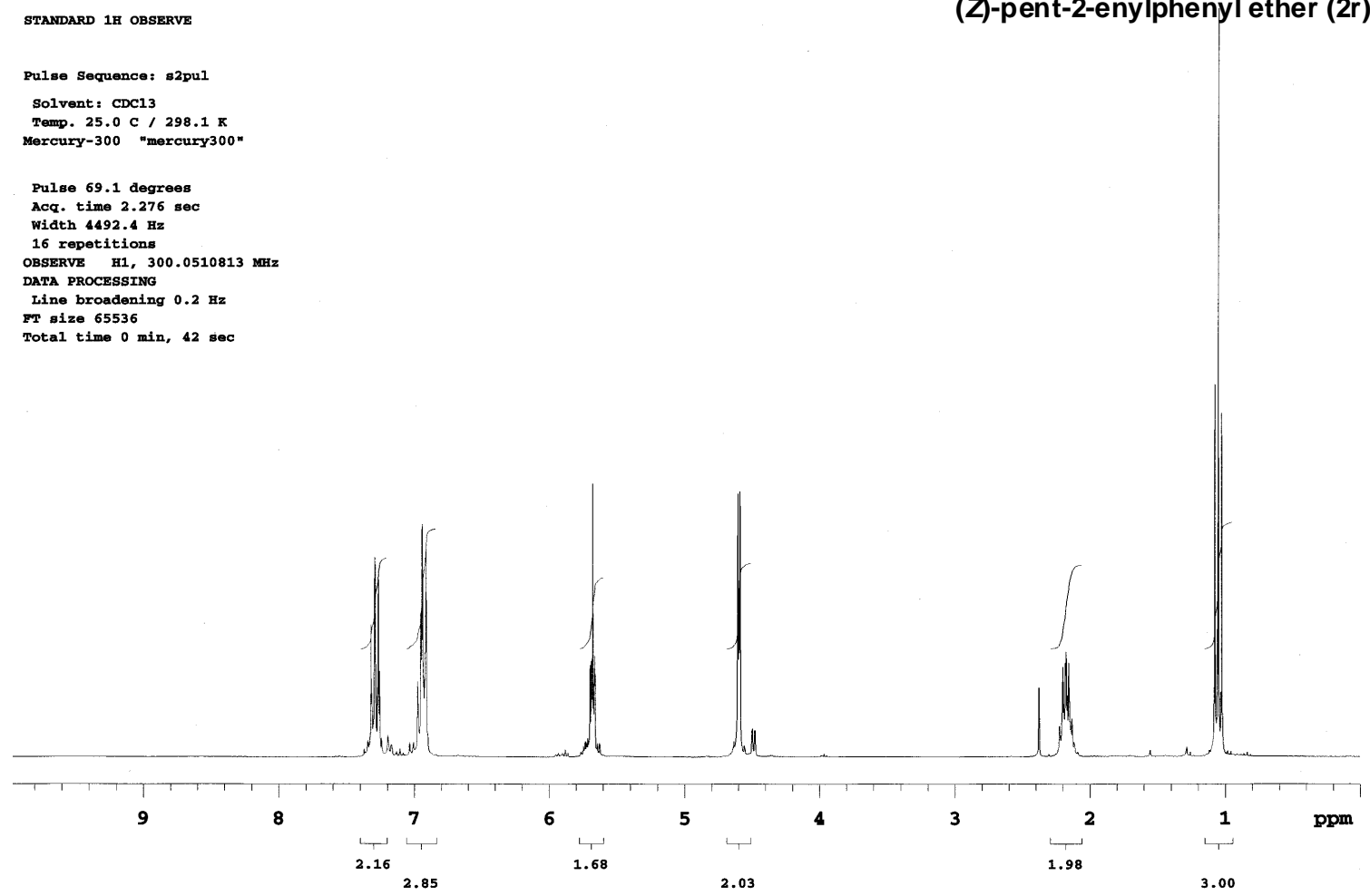

13C OBSERVE

Pulse Sequence: s2pul

Solvent: $\mathrm{CDC} 13$

$25.0 \mathrm{C} / 298.1 \mathrm{k}$

Mercury-300 "mercury300"

Pulse 48.6 degrees

Acq. time 1.000 sec

width $18797.0 \mathrm{~Hz}$

488 repetitions

OBSERVE C13， 75.4478973 MF

DeOOPL $300.0525807 \mathrm{MHz}$

Power 40 as

continuousy on

DATA PROCESSTMG

Line broadening 1.0

Tize 131072

Total time $1 \mathrm{hr}, 16 \mathrm{~min}, 22 \mathrm{sec}$

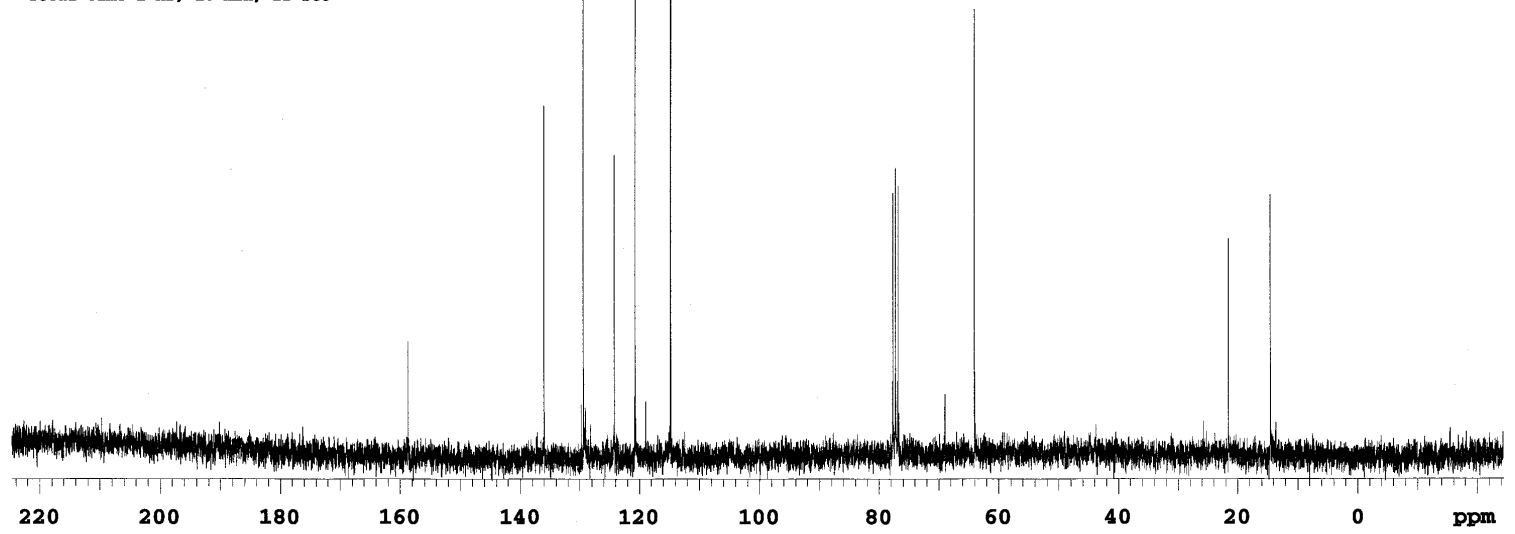




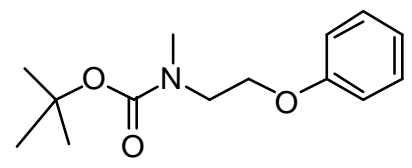

STANDARD 1H OBSERVE

Pu1se Sequence: s2pul

Solvent: $\mathrm{CDCl}$

/ $298.1 \mathrm{~K}$

cury-300 nmercury300"

Pulse 65.6 degrees

Acq. time 2.276 soc

width 4492.4 Hz

OBSERVE H1, 300.0510813 MHz

OBSERVE H1, 30

DATA PROCESSING

7 size 65536

methyl-(2-phen oxyethyl)carbamic acid tert-butyl ester (2u)

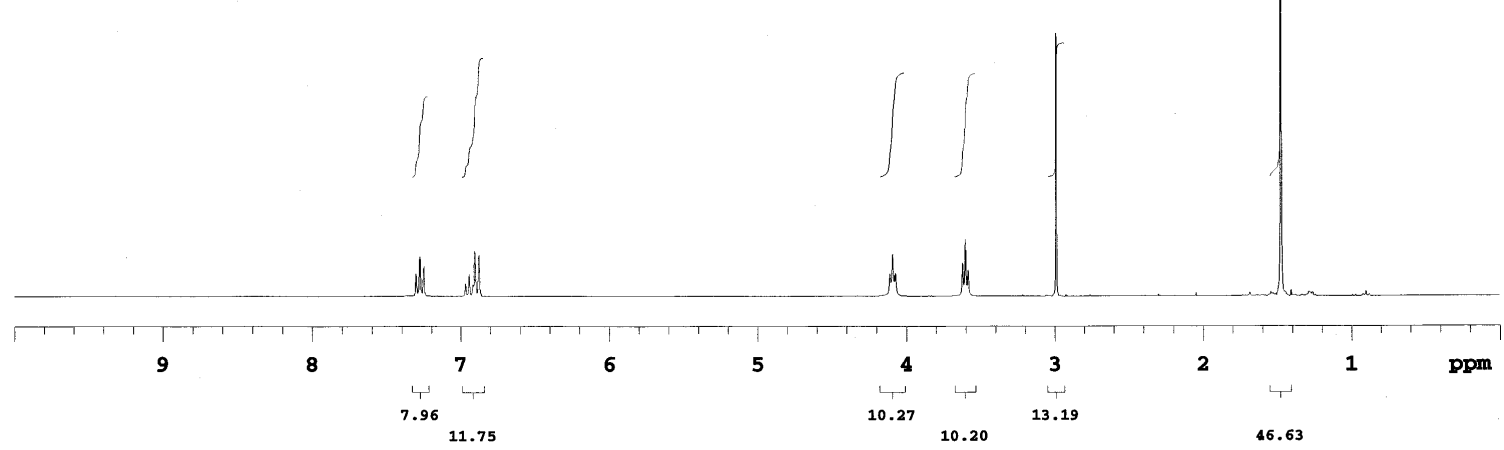

13C OBSERVE

Pu1se Sequence: s2pul

Solvent: CDC13

Temp. $25.0 \mathrm{C} / 298.1 \mathrm{x}$

Mercury-300 "mercury300

Pulse 45.0 degrees

Acq. time $1.000 \mathrm{sec}$

width $18797.0 \mathrm{~Hz}$

800 repetitions

OBSERVE C13, 75.4479008 MH

DECOUPLE H1, $300.0525807 \mathrm{MHZ}$

Power 39 aB

continuously on

WALTZ-16 modulated

DATA PROCESSING

Line broadening $1.0 \mathrm{H}$

T size 131072

Total time $1 \mathrm{hr}, 2 \mathrm{~min}, 5 \mathrm{sec}$

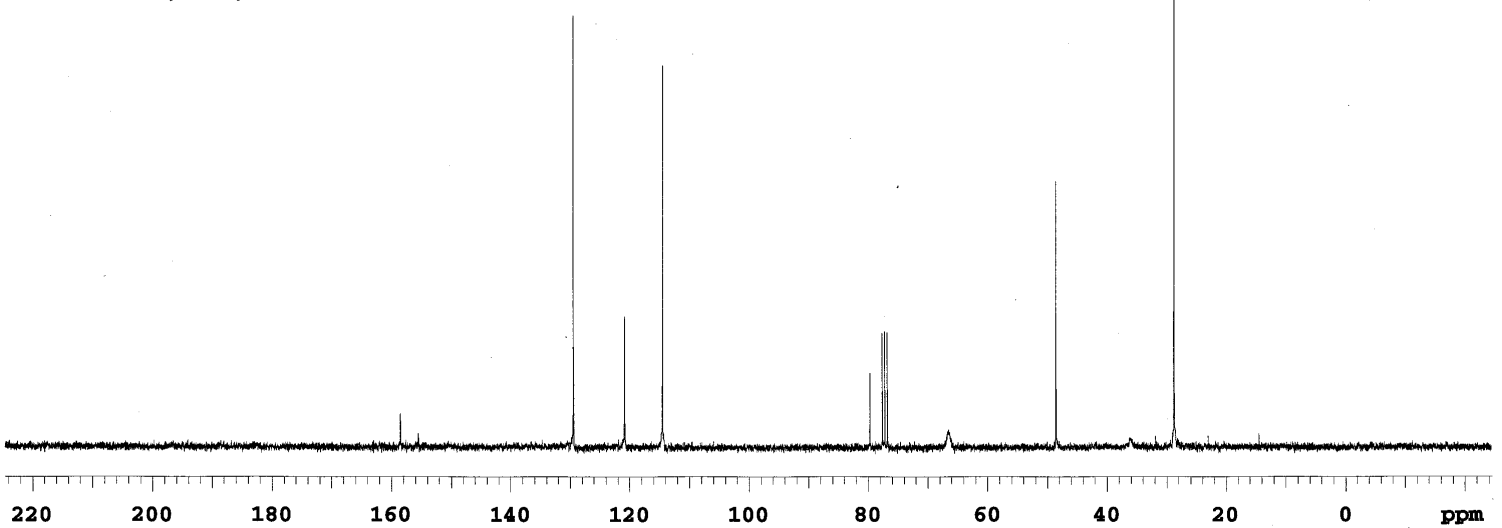



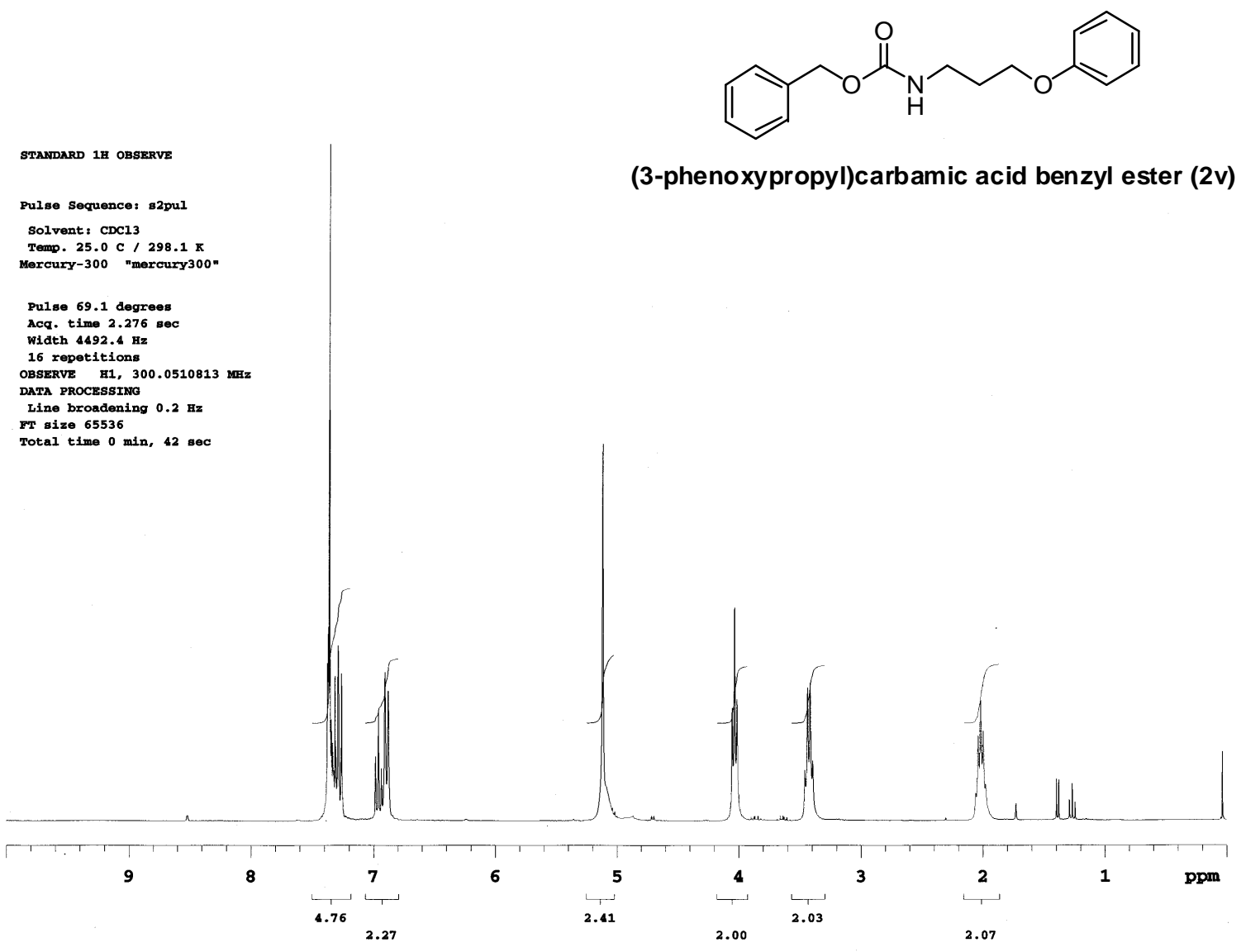

remp. $25.0 \mathrm{C} / 298.1 \mathrm{~K}$

13C OBSERVE

Pulse Sequence: s2pul

Solvent: $\mathrm{CDC13}$

Temp. $25.0 \mathrm{C} / 298.1 \mathrm{~K}$

Mercury-300 mercury300

Pulse 48.6 degrees

width $18797.0 \mathrm{~Hz}$

800 repetitions

OBSERVE C13， 75.4479002 $\mathrm{MBZ}$

DECOUPLE H1, $300.0525807 \mathrm{MHz}$

Power 40 dB

continuously on

WALTZ-16 modulate

DATA PROCESSTNG

Line broadening 1.0

Total time $1 \mathrm{hr}, 2 \mathrm{~min}, 5 \mathrm{sec}$

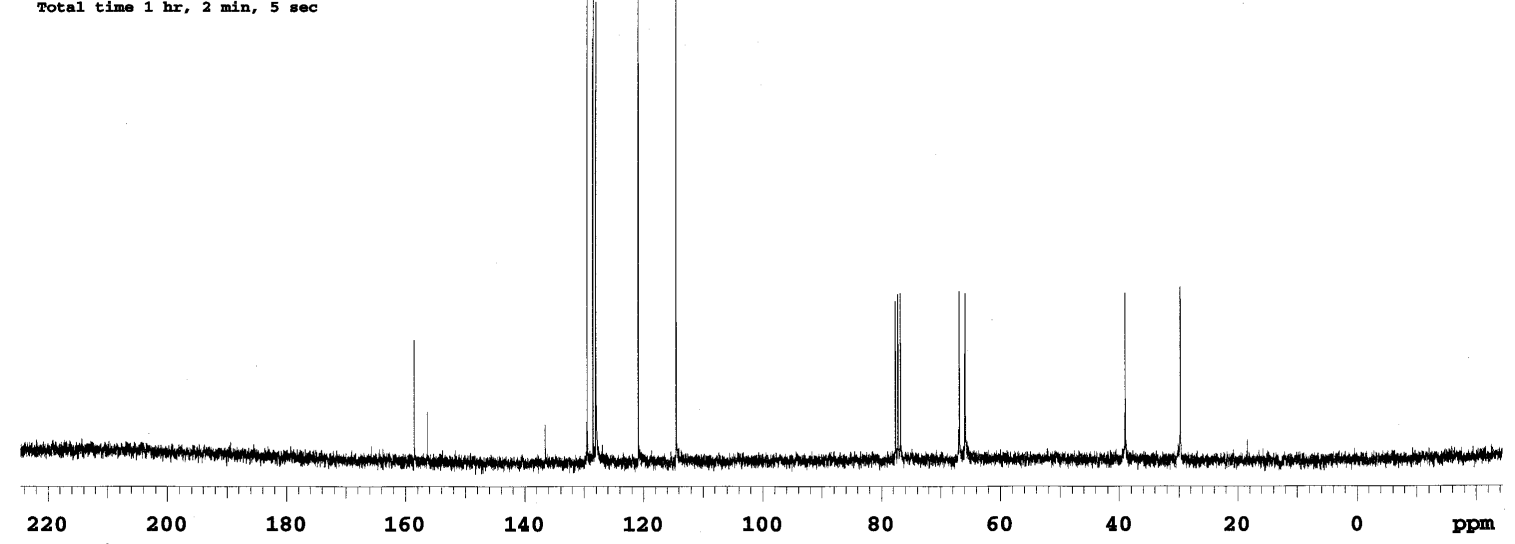



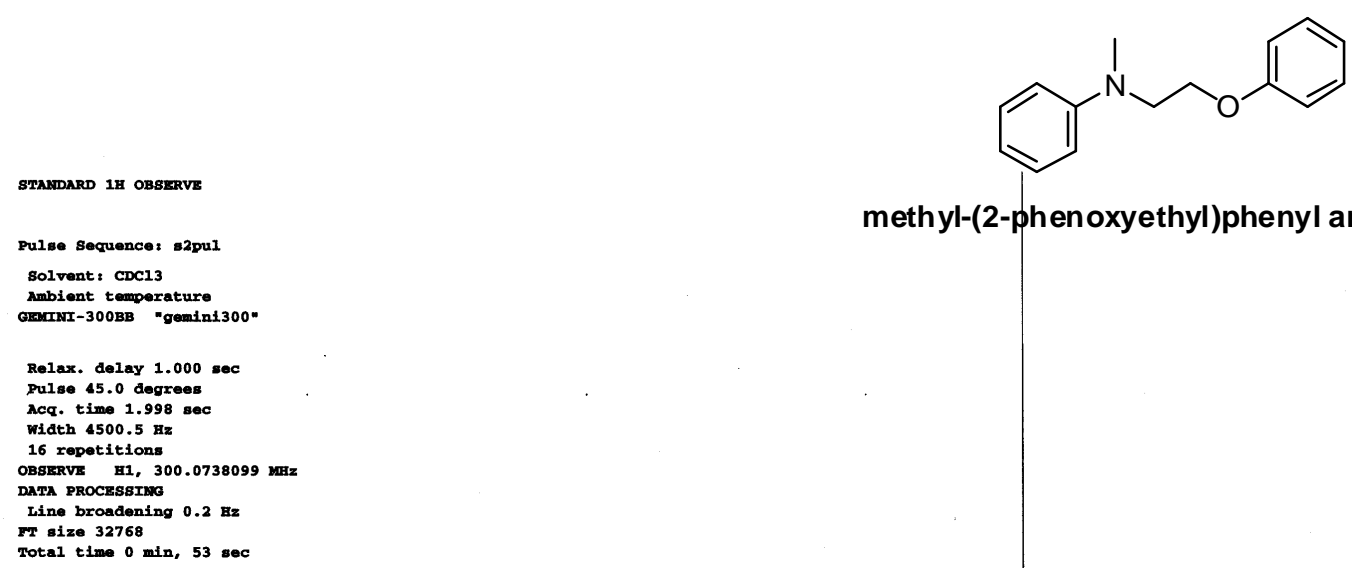

meth yl-(2-phen oxyethyl)phenyl amine (2w)

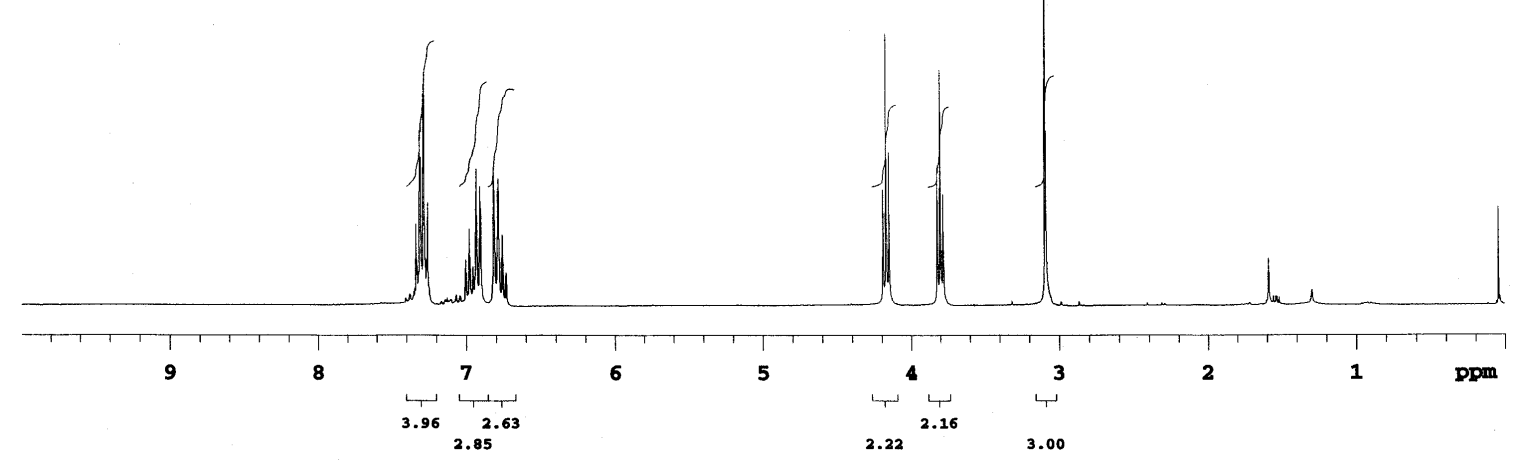

13C OBSERVE

pulse Sequence: \&2pul

Solvent: $\operatorname{CDC13}$

Ambient temperature
GEMTINI-300BB "gemini300"

Pulse 51.1 degrees

Acq. time $1.815 \mathrm{soc}$

wath 18761.7 y

OBSERVE C13, 75.4536093

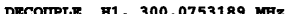

Power 33

continuously on

waLTZ-16 modulated

DATA PROCESSTMG

Line broadening $1.0 \mathrm{~Hz}$

FT size 131072

Total time $40 \mathrm{~min}, 36 \mathrm{sec}$

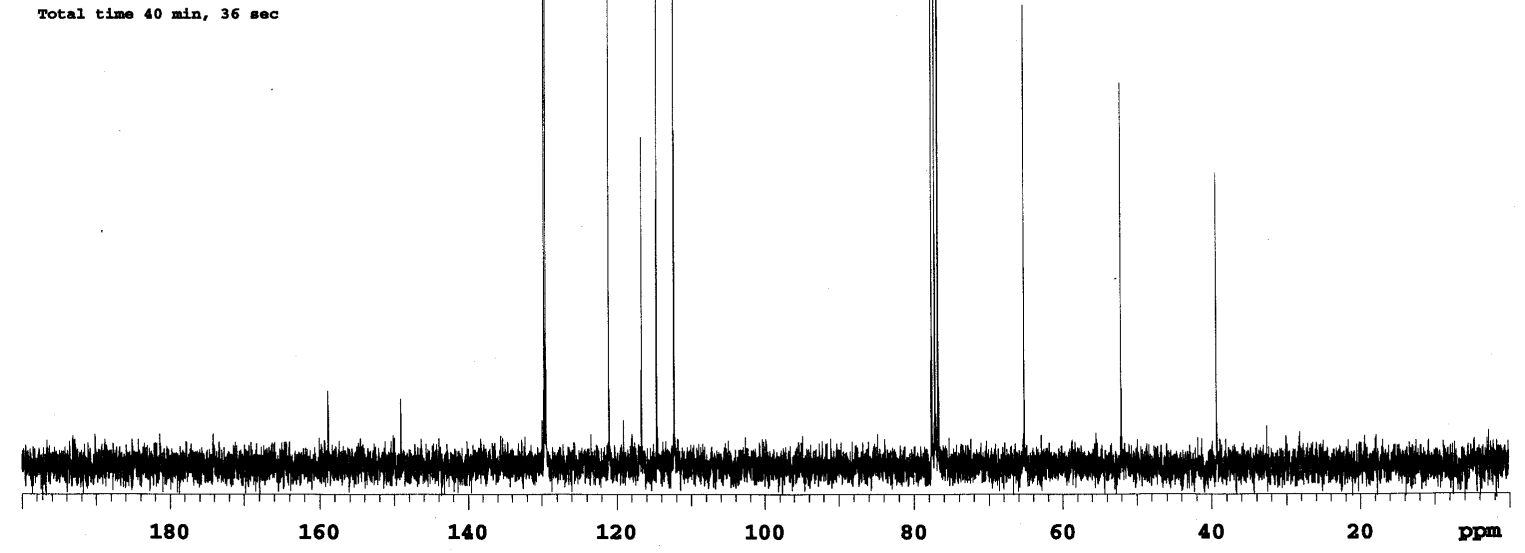



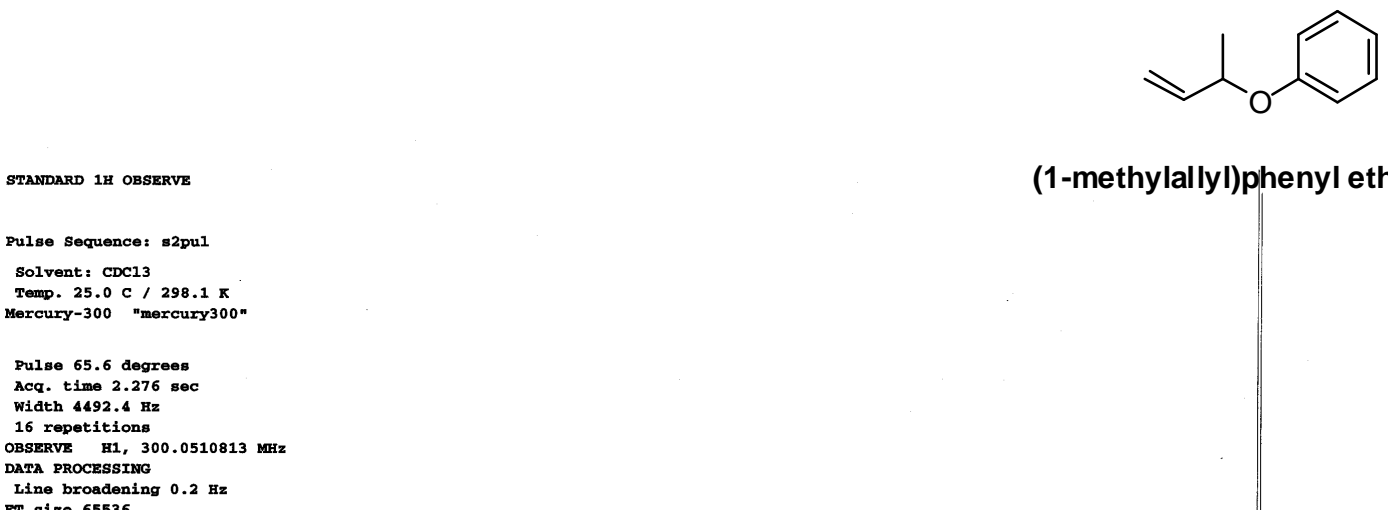

\section{(1-methylal lyl)phenyl ether (2y)}

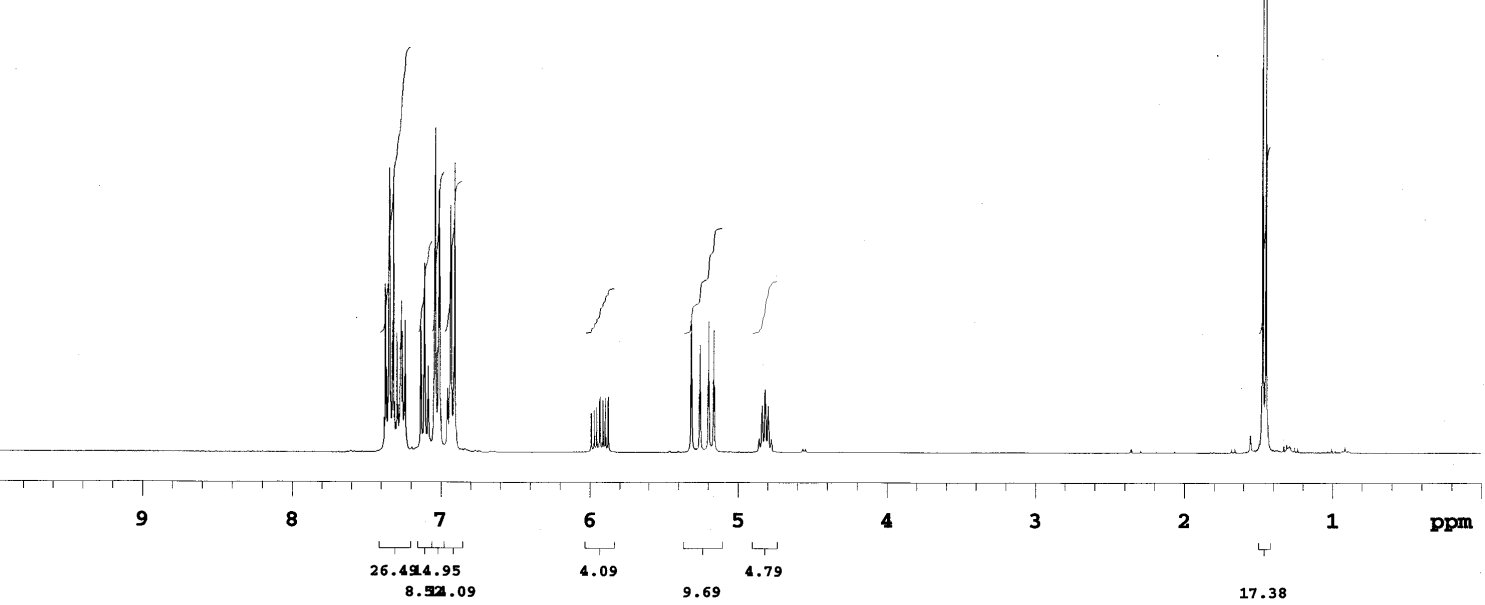

13C OBSERVE

Pulse Sequence: s2pul

Solvent: $\mathrm{CDC13}$

Temp. $25.0 \mathrm{C} / 298.1 \mathrm{~K}$

Mercury-300 "mercury300"

Pulse 45.0 degrees

Acq. time $1.000 \mathrm{sec}$

width $18797.0 \mathrm{~Hz}$

OBSERVE C13， 75.4478968 MHE

DECOUPLE H1, $300.0525807 \mathrm{MHZ}$

Power 39 dB

continuously on

WALTZ-16 modulat

DATA PROCESSING

Line broadening $1.0 \mathrm{~Hz}$

Tize 131072

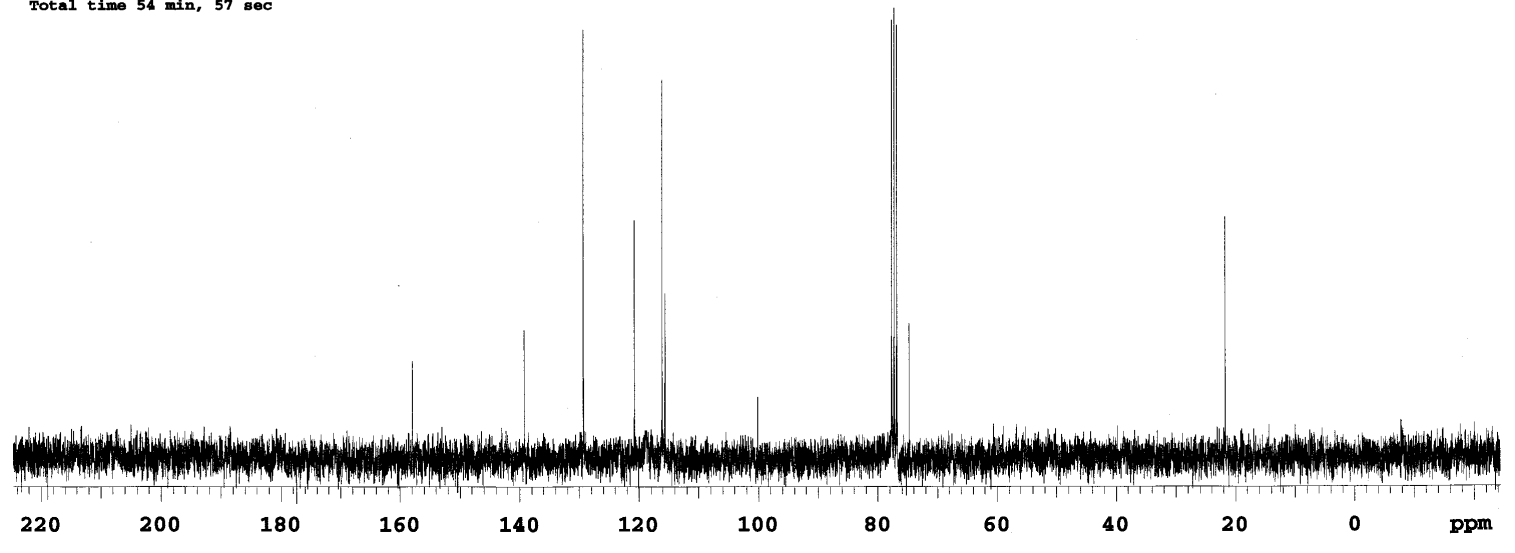




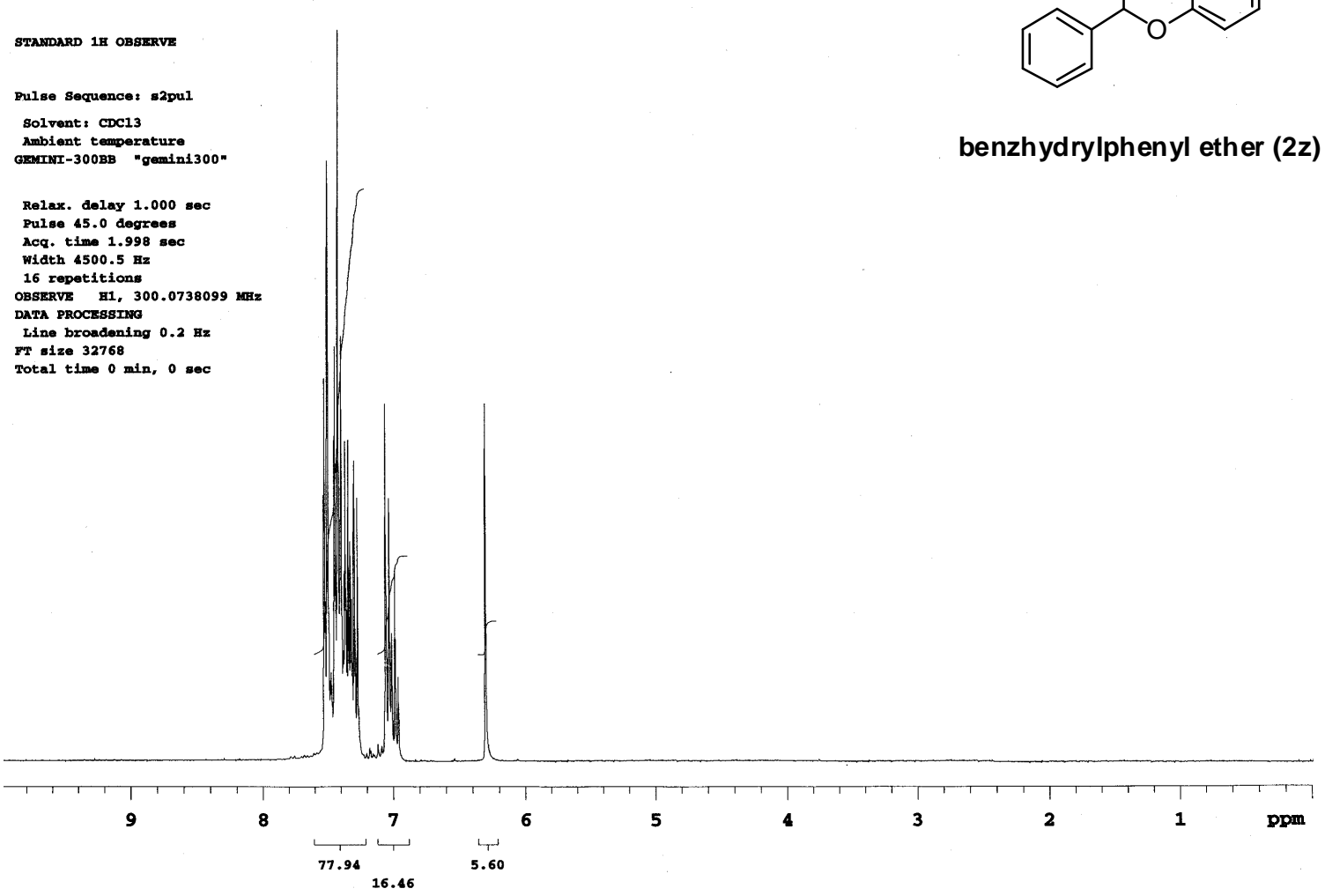

$13 C$ OBSERVE

Pulse Sequence: s2pul

Solvent: CDCl3

GEMTrII-300BB "gemini300"

Pulse 28.6 degrees

Acq. time $1.815 \mathrm{sec}$

width $18761.7 \mathrm{~Hz}$

224 repetitions

OBSERVE C13, 75.4536139 Ma

DECOUPLE H1, 300

Power 33 das

conter

matro

Line broadening

FT size 131072

Total time $49 \mathrm{~min}, 41 \mathrm{sec}$<smiles>c1ccc(OC(c2ccccc2)c2ccccc2)cc1</smiles>

benzhydrylphenyl ether (2z)

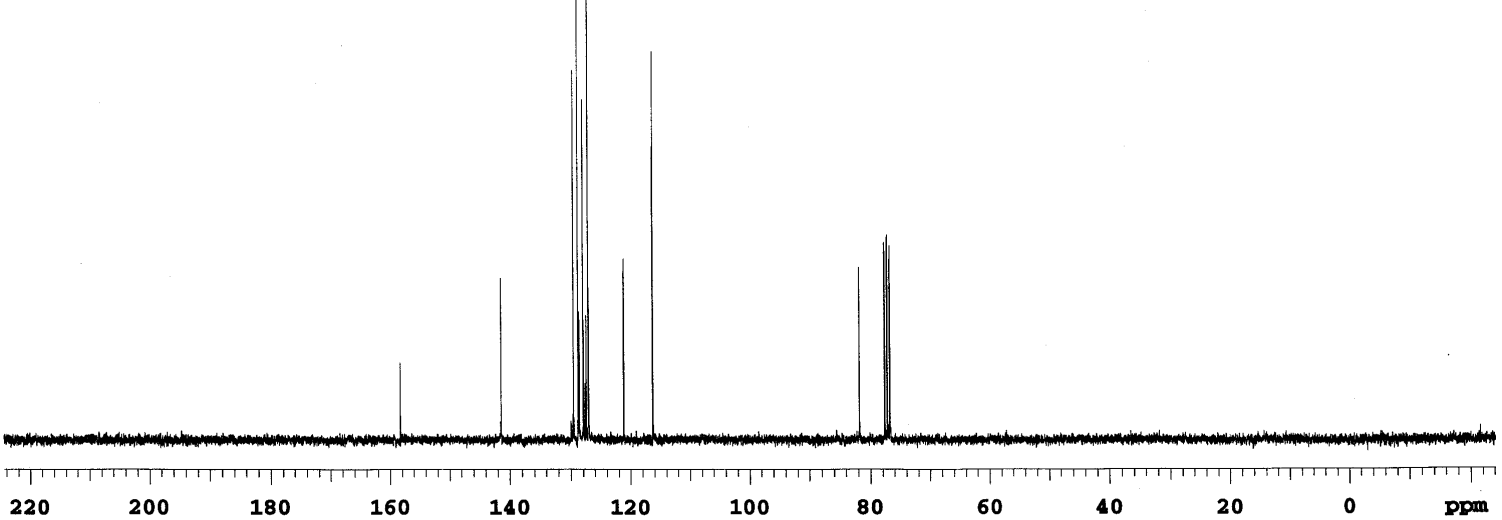


STANDARD 1H OBSERV

Pu1se Sequence: s2pu

Solvent: $\mathrm{CDC1} 3$

Temp. $25.0 \mathrm{C} / 298.1 \mathrm{~K}$

Mercury-300 "mercury300"

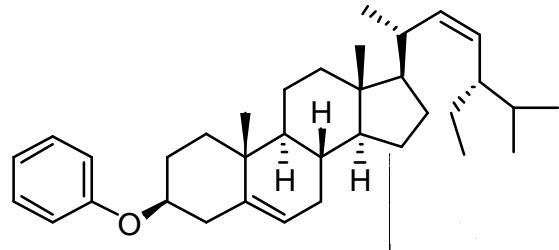

Pulse 65.6 degrees

$2.276 \mathrm{se}$

wiath $4492.4 \mathrm{~Hz}$

OBSERVE H1, 300.0510813 MHz

DATA PROCESSING

Line broadening $0.2 \mathrm{~Hz}$

T size 6553

Total time $0 \mathrm{~min}, 42 \mathrm{sec}$

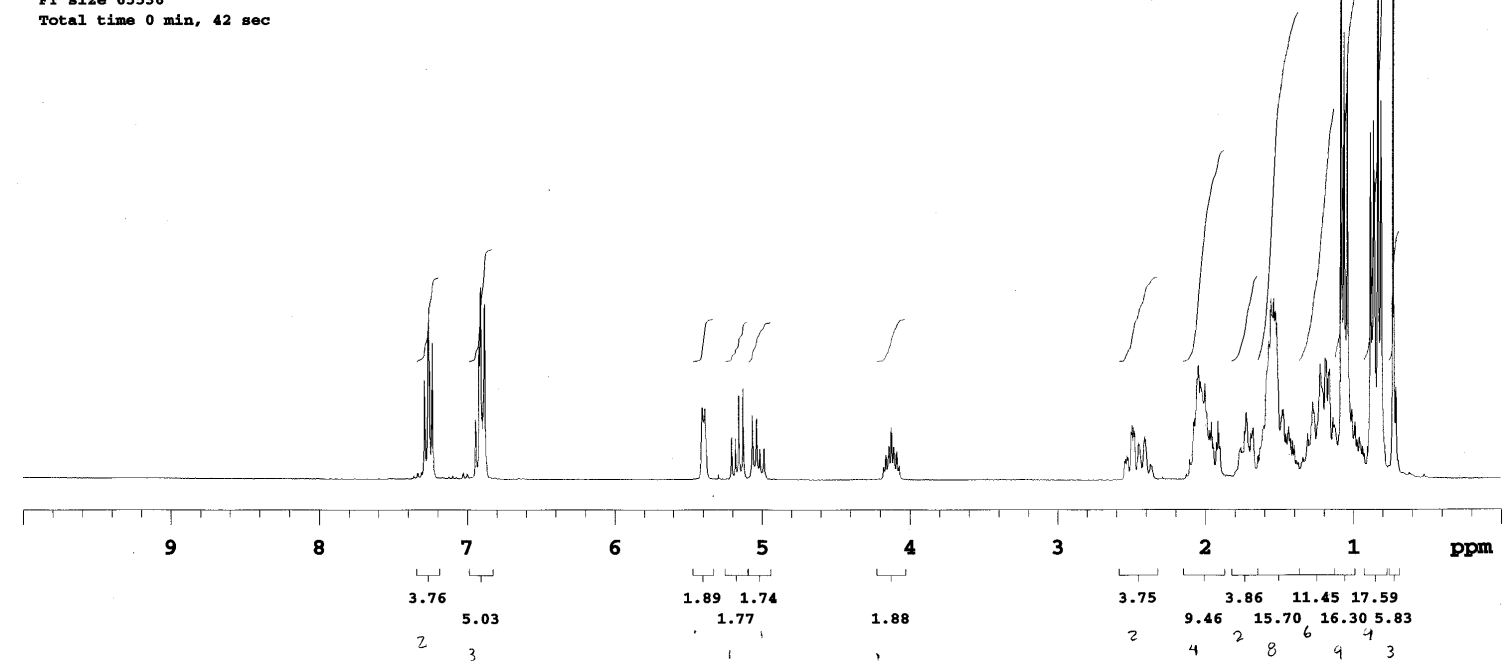

13C OBSERVE

Pulse Sequence: s2pul

Solvent: $\mathrm{CDCl} 13$

Temp. $25.0 \mathrm{C} / 298.1 \mathrm{~K}$

Mercury-300 "mercury300"

Pulse 46.1 degrees

Acq. time $1.000 \mathrm{sec}$

OBSERVE C13， 75.4478956 MHE

DECOUPLE H1, 300.0525807 MHZ

Power 39 dB

continuously on

WALTZ-16 modulated

DATA PROCESSING

Line broadening $1.0 \mathrm{H}$

Total time $1 \mathrm{hr}, 2 \mathrm{~min}, 5 \mathrm{sec}$

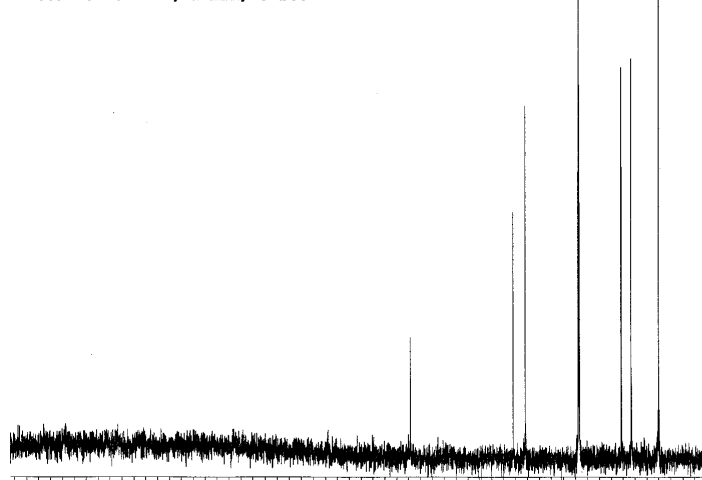




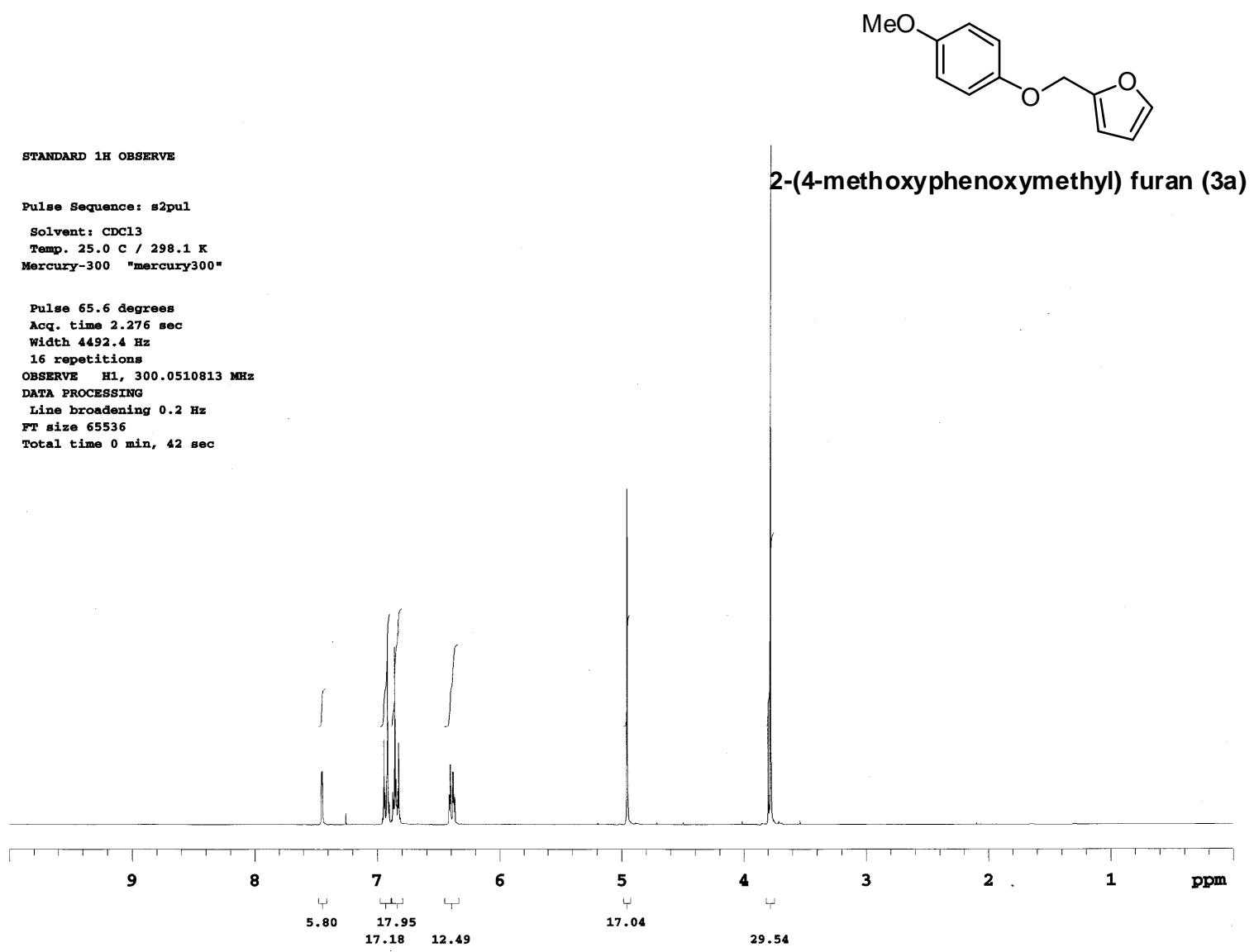

13C OBSERVE

Pulse Sequence: s2pu1

Solvent: $\mathrm{CDC}_{13}$

Temp. $25.0 \mathrm{C} / 298.1 \mathrm{~K}$

Mercury-300 "mercury300"

pulse 45.0 degrees

Acq. time $1.000 \mathrm{Bg}$

488 repetitions

OBSERVE C13, 75.4478999 MHE

DECOUPLE H1, $300.0525807 \mathrm{MHZ}$

Power 39 as

continuously on

waLTZ-16 modulated

DATA PROCESSING

Line broadening $1.0 \mathrm{H}$

FT size 131072

Total time $1 \mathrm{hr}, 2 \mathrm{~min}, 5 \mathrm{sec}$

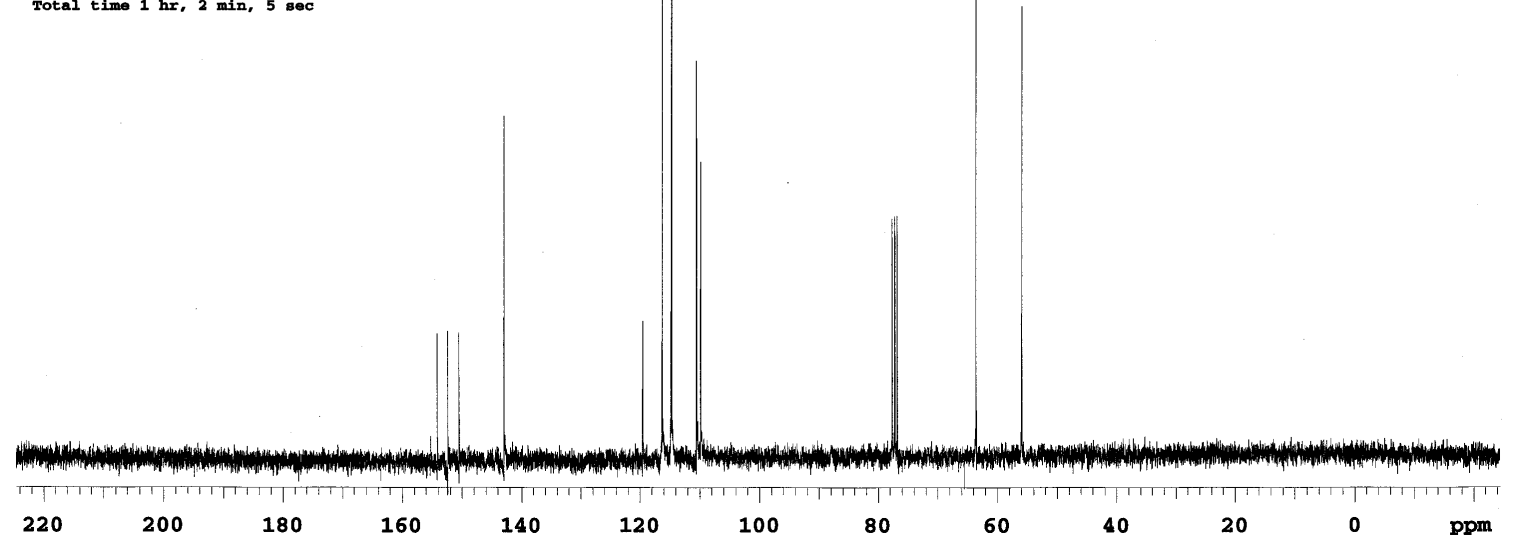




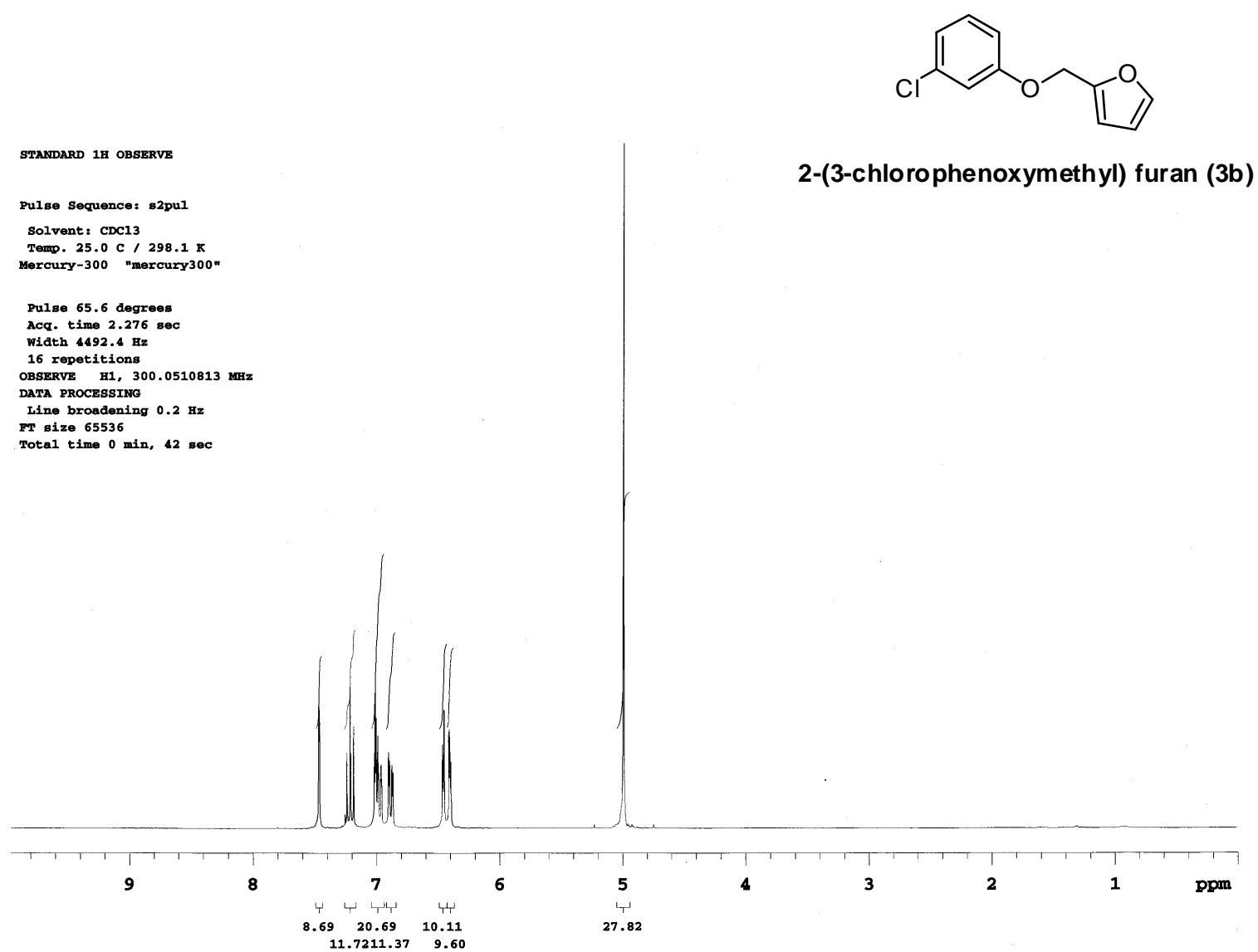

13C OBSERVE

Pulse Sequence: s2pul

Solvent: $\mathrm{CDC13}$

Temp. $25.0 \mathrm{C} / 298.1 \mathrm{~K}$

Mercury-300 "mercury300

Pulse 46.1 degrees

Acq. time $1.000 \mathrm{sec}$

width $18797.0 \mathrm{~Hz}$

352 repetitions

OBSERVE C13， 75.4479016 MHE

DECOUPLE H1, $300.0525807 \mathrm{MHz}$

Power 39 dB

continuously on

WALTZ-16 modulated

DATA PROCESSING

Line broadening $1.0 \mathrm{~Hz}$

Total time $1 \mathrm{hx}, 2 \mathrm{~min}, 5 \mathrm{sec}$

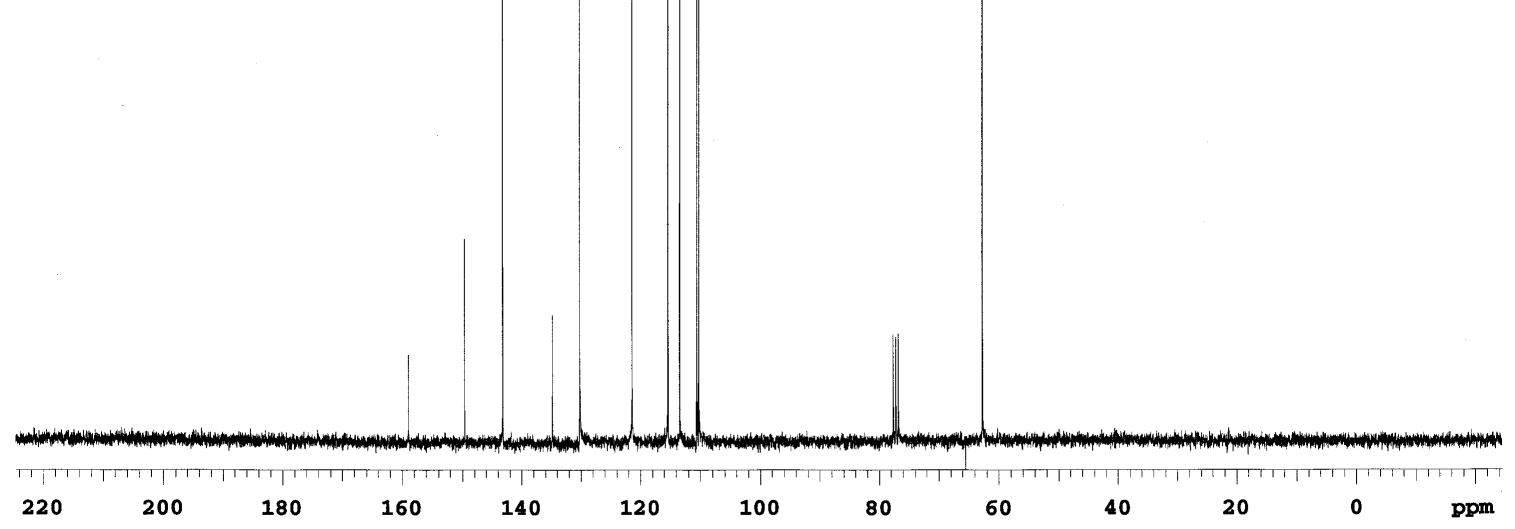



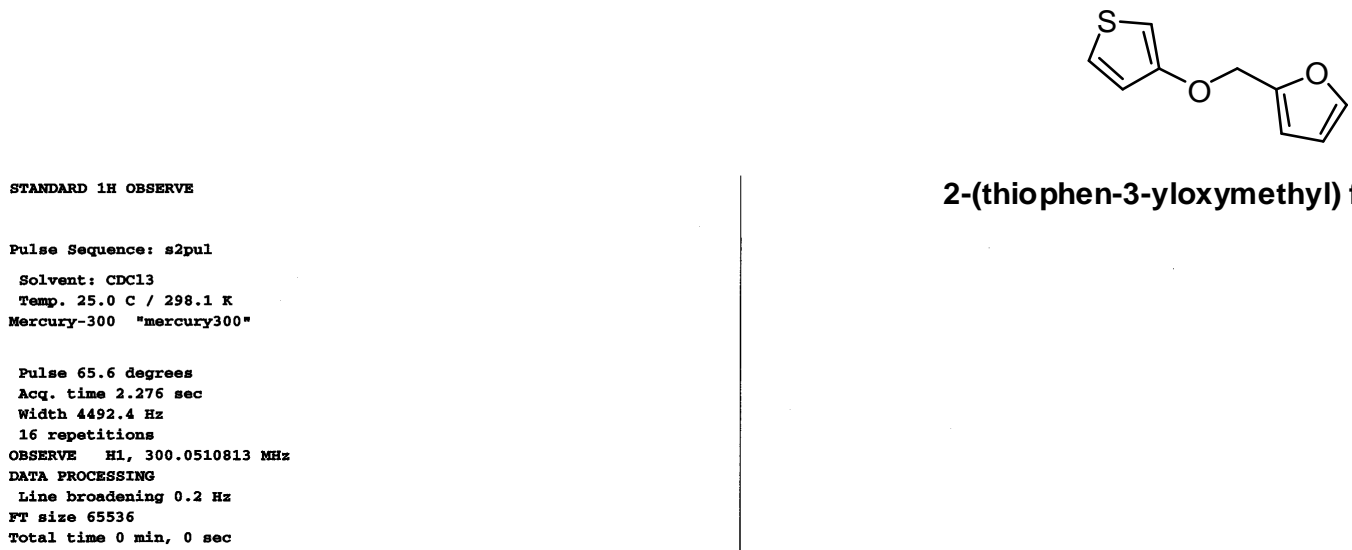

2-(thiophen-3-yloxymethyl) furan (3c)

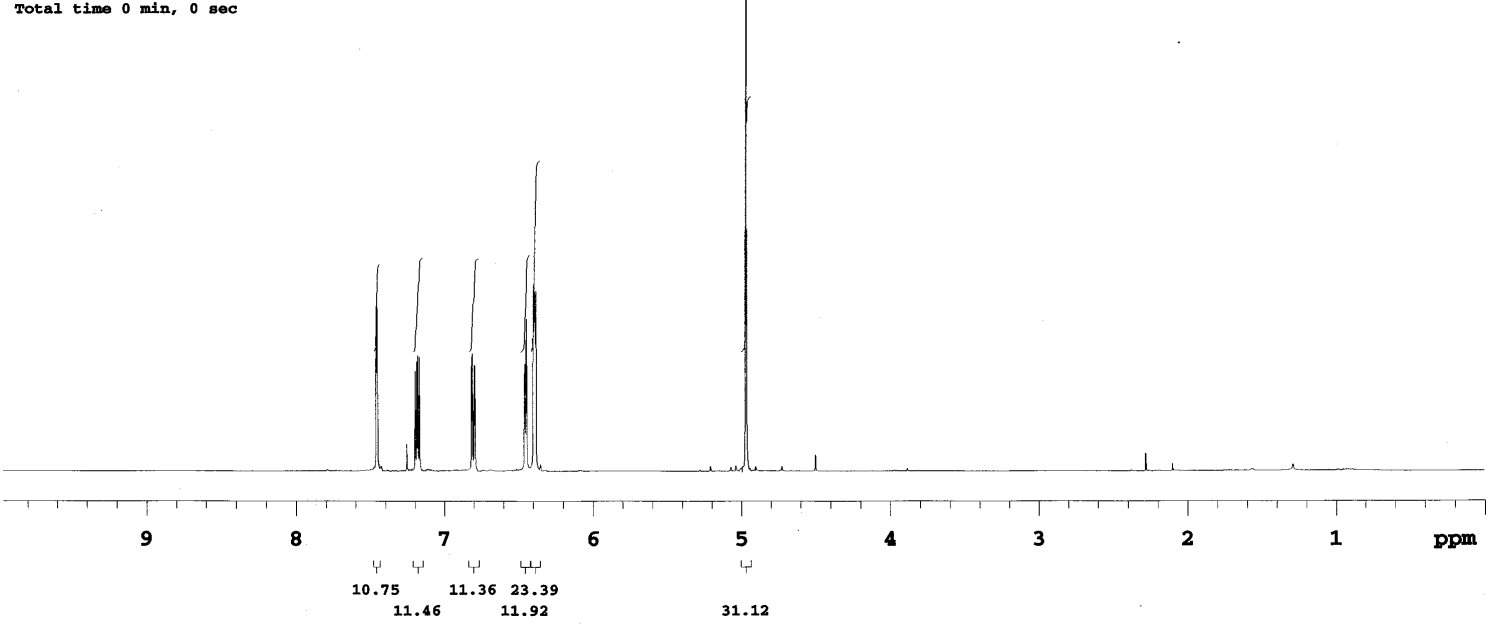

13C OBSERVE

Pulse Sequence: s2pul

Solvent: $\mathrm{CDC}^{3} 3$

Temp. $25.0 \mathrm{C} / 298.1 \mathrm{k}$

Pulse 45.0 degrees

Acg. time $1.000 \mathrm{sec}$

width $18797.0 \mathrm{~Hz}$

ORSERVE C13, 75.4478994 MFE

OBSERVE C13, 75.4478994 MHZ

Cover.

continuousiy

WALTZ-16 modulate

DATA PROCESSING

Line broadening $1.0 \mathrm{~Hz}$

FT size 131072

Total time $1 \mathrm{hr}, 2 \mathrm{~min}, 5 \mathrm{sec}$
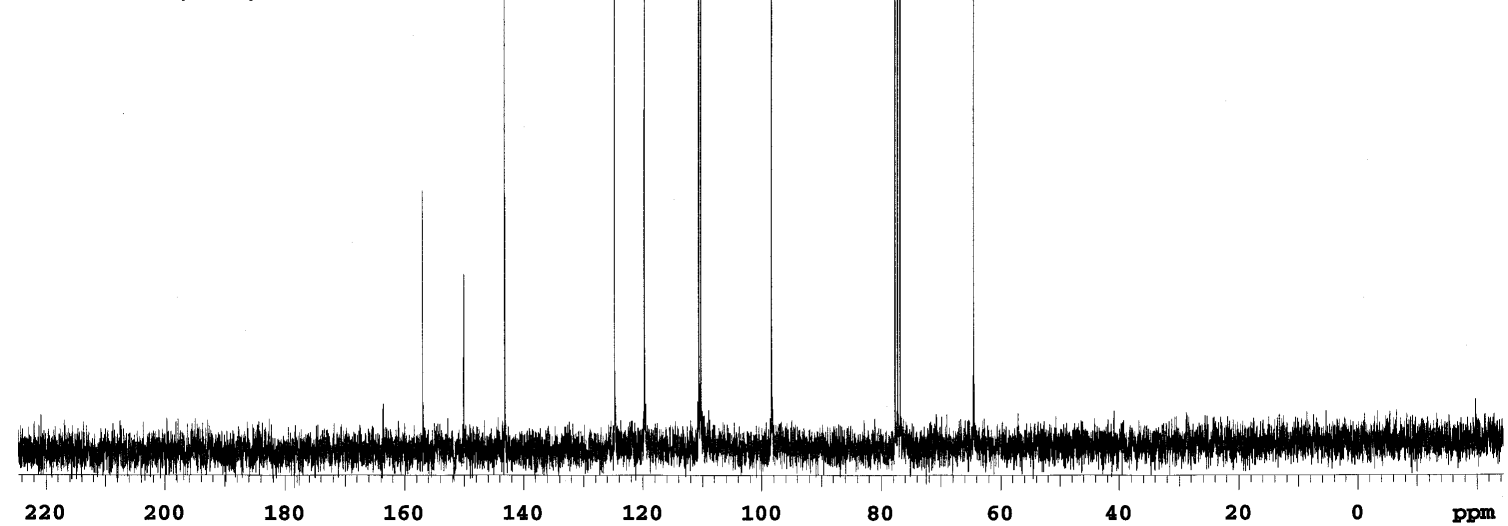


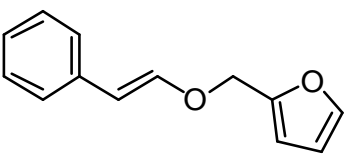

STANDARD 1H OBSERV

Pulse Sequence: s2pul

Solvent: CDC13

Temp. $25.0 \mathrm{C} / 298.1 \mathrm{x}$

Relax. delay $5.000 \mathrm{sec}$

Pulse 83.9 degrees

Acq. time $2.276 \mathrm{sec}$

width 4492.4 Hz

16 repetitions

OBSERVE H1, 300.0510813 MH

DATA PROCESSIMG

Line broadening $0.2 \mathrm{~Hz}$

Fis size 65536

Total time $2 \mathrm{~min}, 2 \mathrm{sec}$

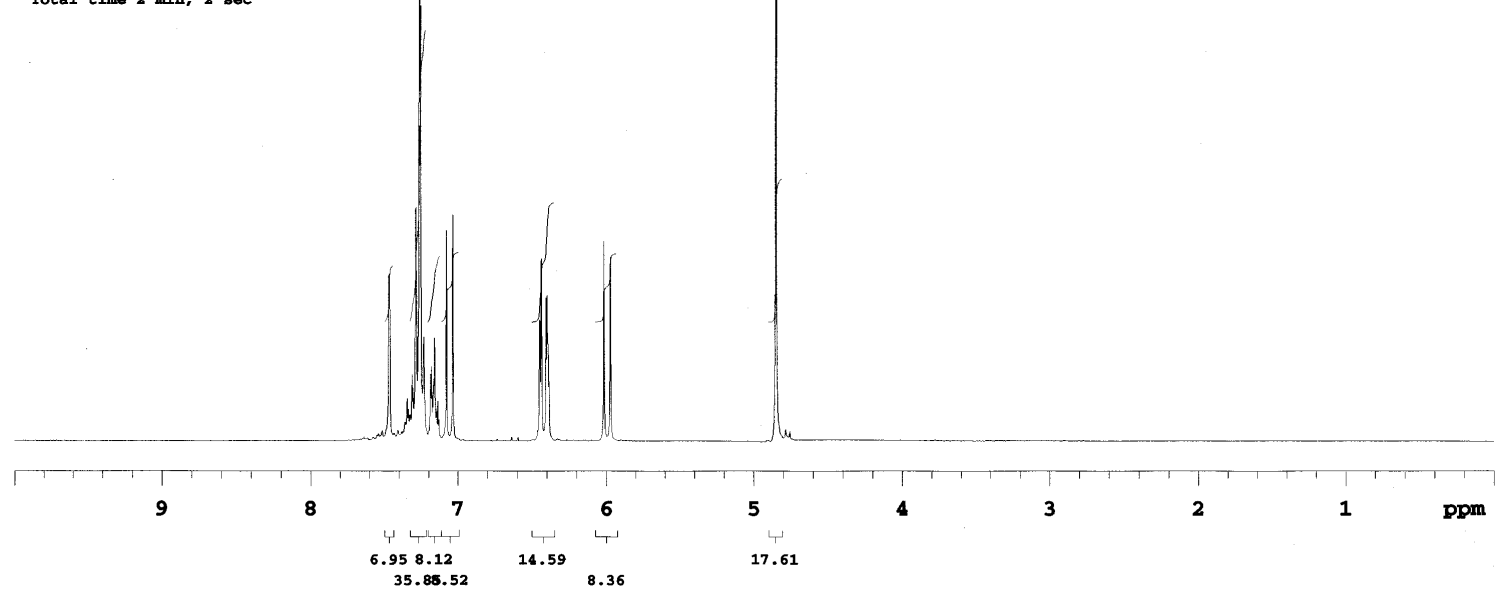

13C OBSERVE

Pulse Sequence: 82pul

Solvent: CDC13

Temp. $25.0 \mathrm{C} / 298.1 \mathrm{~K}$

Mercury-300 "mercury300

Pulse 46.1 degrees

width $18797.0 \mathrm{~Hz}$

272 repetitions

OBSERVE C13, 75.4478994 MHZ

DECOUPLE H1, 300.0525807 MT:

Power $39 \mathrm{~dB}$

continuously on

WALTz-16 modulate

DATA PROCESSING

Line broadening $1.0 \mathrm{~Hz}$

FT size 13107

Total time $1 \mathrm{hr}, 16 \mathrm{~min}, 22 \mathrm{sec}$

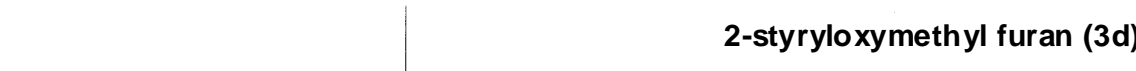

8.36

pm

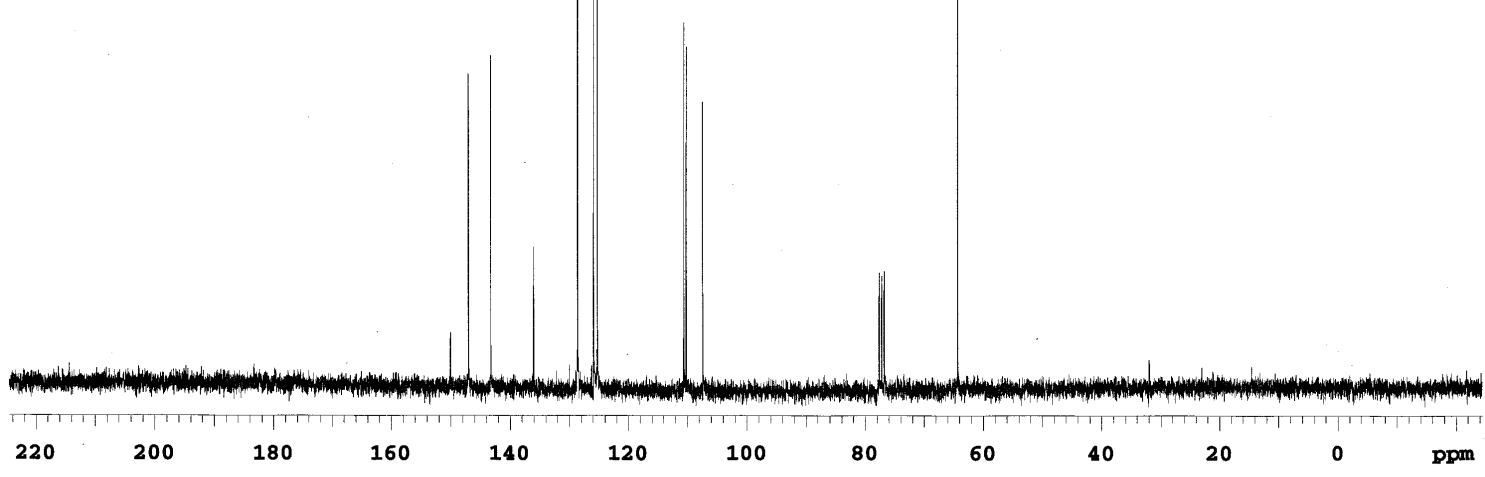




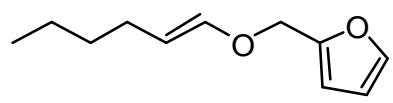

2-hex-1-enyloxymethyl) furan (3e)

STANDARD 1H OBSERVE

Pu1se sequence: s2pul

Solvent: $\mathrm{CDC}_{13}$

Temp. $25.0 \mathrm{C} / 298.1 \mathrm{~K}$

Mercury-300 "mercury 300 "

Pulse 65.6 degrees

Acq. time $2.276 \mathrm{sec}$

idth $4492.4 \mathrm{~Hz}$

OBSERVE H1, 300.0510813 MF

DATA PROCESSING

Line broadening $0.2 \mathrm{~Hz}$

Total time 0 min, $42 \mathrm{sec}$

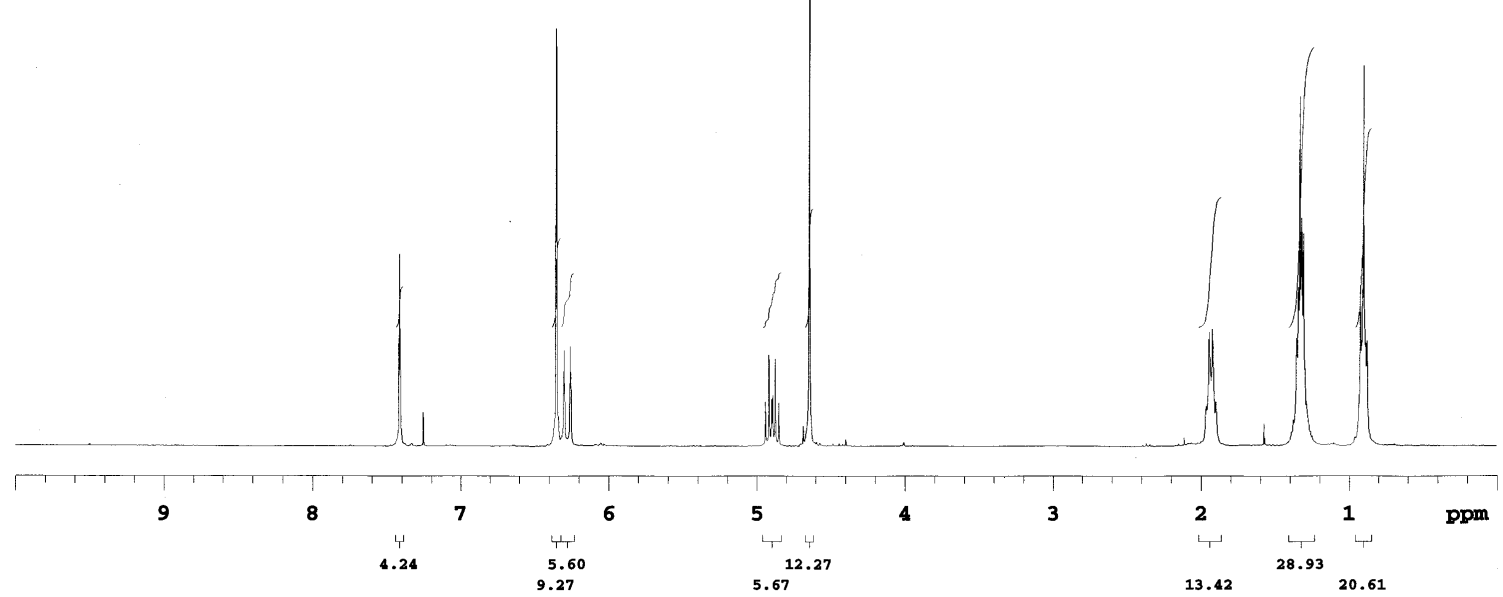

13C OBSERVE

Pu1se Sequence: s2pul

Solvent: $\mathrm{CDC}_{13}$

Temp. $25.0 \mathrm{C} / 298.1 \mathrm{~K}$

ercury-300 "mercury300"

Pu1se 46.1 degrees

Acq. time 1.000 sec

Width $18797.0 \mathrm{~Hz}$

416 repetitions

OBSERVE C13, 75.4478973 MHz

Cl, 300.05

Power 39 dB

WALTZ-16 modulated

DATA PROCESSING

Line broadenin

Total time $1 \mathrm{hr}, 2 \mathrm{~min}, 5 \mathrm{sec}$
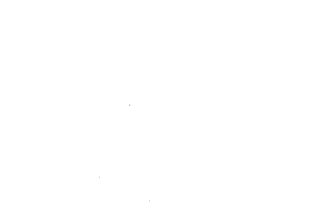

(1)

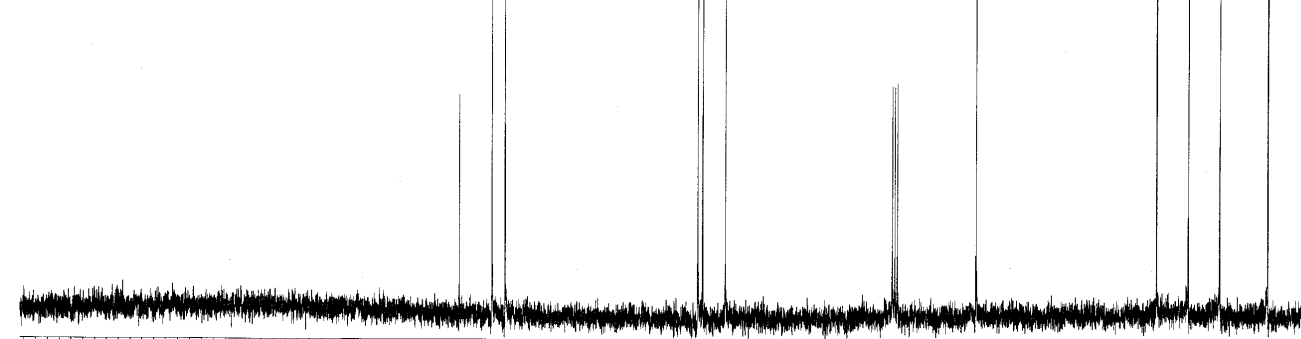

40

20

o ppm 